\title{
NEGATIVE POWERS OF INTEGRATED PROCESSES
}

\author{
Neslihan Sakarya \\ University of Essex \\ ROBERT M. DE JONG \\ Ohio State University
}

\begin{abstract}
This paper derives the limit distribution of the rescaled sum of the absolute value of an integrated process with continuously distributed innovations raised to a negative power less than -1 , and of the analogous statistic that is obtained using the same function of an integrated process but only considering positive values of the integrated process. We show that the limit behavior of this statistic is determined by the values of the integrated process that are closest to 0 , and find the limit behavior of the values of the integrated process that are closest to 0 .
\end{abstract}

\section{INTRODUCTION}

Pötscher (2013) studied statistics of the form $\sum_{t=1}^{n}\left|x_{t}\right|^{-q}$ for $q>1$ and an integrated process $x_{t}$ satisfying some regularity conditions, and established the order of magnitude of such statistics. Among other results, Pötscher (2013) showed that $n^{-q / 2} \sum_{t=1}^{n}\left|x_{t}\right|^{-q}=O_{p}(1)$ for $q>1$. This paper will show that $n^{-q / 2} \sum_{t=1}^{n}\left|x_{t}\right|^{-q}$ converges in distribution for $q>1$ under regularity conditions, and provides a characterization of the limit distribution. Therefore, the rate established in Pötscher (2013) was indeed the best possible one. Note that in order to prevent a division by zero issue, a regularity condition ensuring the continuity of the distribution of $x_{t}$ is needed to analyze such statistics.

As Pötscher (2013) noted, the asymptotic behavior of expressions of the form $r_{n} \sum_{t=1}^{n} f\left(k_{n} x_{t}\right)$, for deterministic sequences $r_{n}$ and $k_{n}$, has been the subject of a number of articles in recent years. In Econometrics, Park and Phillips (1999) started the interest in this topic. The work of Pötscher (2004) and de Jong (2004) contained results that showed that for $r_{n}=n^{-1}, k_{n}=n^{-1 / 2}, n^{-1 / 2} x_{[r n]} \Rightarrow \lambda W(r)$ for $r \in[0,1], W(\cdot)$ Brownian motion, $\lambda^{2}$ a variance parameter, and $f(\cdot)$ absolutely integrable on finite intervals, the limit $\int_{0}^{1} f(\lambda W(r)) d r$ can be found under regularity conditions. Therefore, because for $0<q<1,|x|^{-q}$ satisfies $\int_{a}^{b}|x|^{-q} d x<\infty$ for any $a, b \in \mathbb{R}, a \leq b$, it follows that $n^{-1+q / 2} \sum_{t=1}^{n}\left|x_{t}\right|^{-q} \stackrel{d}{\longrightarrow} \int_{0}^{1}|\lambda W(r)|^{-q} d r$. This result does not follow immediately from the continuous mapping, because the pole

Author correspondence to Neslihan Sakarya, Department of Economics, University of Essex, Colchester, UK; e-mail: ns17003@essex.ac.uk.

(C) The Author(s), 2021. Published by Cambridge University Press. This is an Open Access article, distributed under the terms of the Creative Commons Attribution-NonCommercial-NoDerivatives licence (http://creativecommons.org/licenses/by-nc-nd/4.0/), which permits non-commercial re-use, distribution, and reproduction in any medium, provided the original work is unaltered and is properly cited. The written permission of Cambridge University Press must be obtained for commercial re-use or in order to create a 
at 0 renders the mapping noncontinuous. Such results however are not informative about the case where $f(\cdot)$ has a nonintegrable pole, such as $f(x)=|x|^{-q}$ for $q \geq 1$.

For the case $q=1$, Pötscher (2013) showed that $n^{-1 / 2}(\log (n))^{-1} \sum_{t=1}^{n}\left|x_{t}\right|^{-1}=$ $O_{p}(1)$ under regularity conditions, and Michel and de Jong (2020) showed that

$n^{-1 / 2}(\log (n))^{-1} \sum_{t=1}^{n}\left|x_{t}\right|^{-1} \stackrel{d}{\longrightarrow} 2 \lambda^{-1}|Z|$

under regularity conditions, where $Z \sim N(0,1)$.

Note that the above results all have one-sided equivalents that are obtained by only summing over the values of $t$ for which $x_{t}$ is positive or negative. Other papers considering statistics of the form $r_{n} \sum_{t=1}^{n} f\left(k_{n} x_{t}\right)$ are Borodin and Ibragimov (1995), Jeganathan (2004), de Jong and Wang (2005), Berkes and Horváth (2006), and Christopeit (2009).

The plan of this paper is as follows. Section 2 outlines the idea of the proof of the main result. In Section 3, we first set out to find convergence in distribution results for the occupation times for small intervals. Note that Akonom (1993, Thm. 2 and Lem. 1) established results for occupation times of the integrated process, but this author's results are not sufficiently tailored to the "small" interval situation to be of use here. We apply these results to show convergence results for $\min _{\left\{t: 1 \leq t \leq n, x_{t}>0\right\}} x_{t}$ and $\min _{1 \leq t \leq n}\left|x_{t}\right|$. In Section 4, we consider multivariate convergence in distribution results for occupation times and convergence results for the order statistics of $\left|x_{t}\right|$ and the positive values of $x_{t}$. Section 5 then derives the limit distribution for the statistics of $n^{-q / 2} \sum_{t=1}^{n}\left|x_{t}\right|^{-q}$ and $n^{-q / 2} \sum_{t=1}^{n} x_{t}^{-q} I\left(x_{t}>0\right)$ for $q>1$. We conclude with Section 6, where we give simulation results for the distributions of $\min _{\left\{t: 1 \leq t \leq n, x_{t}>0\right\}} x_{t}, \min _{1 \leq t \leq n}\left|x_{t}\right|, n^{-1} \sum_{t=1}^{n} x_{t}^{-2} I\left(x_{t}>0\right)$, and $n^{-1} \sum_{t=1}^{n} x_{t}^{-2}$.

\section{MAIN IDEA OF THE PROOF}

Let $x_{t}$ be an integrated process that is a recurrent random walk with i.i.d. innovations. In this paper, our approach is to write

$n^{-q / 2} \sum_{t=1}^{n}\left|x_{t}\right|^{-q}=\sum_{t=1}^{n}\left|n^{1 / 2} x_{t}\right|^{-q}=\sum_{t=1}^{n} Z_{n t}^{-q}$,

where $Z_{n t}$ is the $t$ th smallest value of $n^{1 / 2}\left|x_{t}\right|, t=1, \ldots, n$. We then show the joint convergence of $\left(Z_{n 1}, \ldots, Z_{n m}\right)^{\prime}$ for any integer $m$, and prove that $n^{-q / 2} \sum_{t=1}^{n}\left|x_{t}\right|^{-q}$ is asymptotically close to $\sum_{t=1}^{m} Z_{n t}^{-q}$. We find the limit distribution of $n^{-q / 2} \sum_{t=1}^{n}\left|x_{t}\right|^{-q}$ based on those results. For $n^{-q / 2} \sum_{t=1}^{n} x_{t}^{-q} I\left(x_{t}>0\right)$, the reasoning is similar, by noting that

$n^{-q / 2} \sum_{t=1}^{n} x_{t}^{-q} I\left(x_{t}>0\right)=\sum_{t=1}^{n}\left(n^{1 / 2} x_{t}\right)^{-q} I\left(x_{t}>0\right)=\sum_{t=1}^{M_{n}} Y_{n t}^{-q}$, 
where $M_{n}$ is the number of positive $x_{t}$ and $Y_{n t}$ is the $t$ th smallest positive value of $n^{1 / 2} x_{t}, t=1, \ldots, n$.

We will first focus on the case $m=1$ and $Z_{n 1}=n^{1 / 2} \min _{1 \leq t \leq n}\left|x_{t}\right|$. Note that of course, from the functional central limit theorem it follows that

$n^{-1 / 2} \min _{1 \leq t \leq n}\left|x_{t}\right| \stackrel{d}{\longrightarrow} \inf _{r \in[0,1]}|W(r)|=0$

but beyond that, the functional central limit theorem is not informative about $\min _{1 \leq t \leq n}\left|x_{t}\right|$.

The behavior of $n^{1 / 2} \min _{1 \leq t \leq n}\left|x_{t}\right|$, but also more general order statistics of this type, can be related to occupation times of integrated processes for small intervals, where "small" here means that the width of the interval is $O\left(n^{-1 / 2}\right)$. Define $S_{n}(y)=$ $\sum_{t=1}^{n} I\left(\left|x_{t}\right| \leq y n^{-1 / 2}\right)$. Then for all $y \in \mathbb{R}$,

$P\left(Z_{n 1} \leq y\right)=P\left(S_{n}(y)>1 / 2\right)$.

If we can now show the convergence in distribution of $S_{n}(y)$ to some limit $S(y)$ for all $y \in \mathbb{R}$, by noting that $1 / 2$ is necessarily a continuity point of the distribution of $S(y)$ because the distribution of $S(y)$ will only put probability mass on the integers, we also find

$\lim _{n \rightarrow \infty} P\left(Z_{n 1} \leq y\right)$

A similar argument can be used for $Y_{n 1}=n^{1 / 2} \min _{\left\{t: 1 \leq t \leq n, x_{t}>0\right\}} x_{t}$. Defining $R_{n}(y)=$ $\sum_{t=1}^{n} I\left(0<x_{t} \leq y n^{-1 / 2}\right)$ we can also note that for all $y \in \mathbb{R}$,

$P\left(Y_{n 1} \leq y\right)=P\left(R_{n}(y)>1 / 2\right)$.

Therefore, the next section will first establish results on the limit behavior of the occupation times for small intervals.

\section{OCCUPATION TIMES FOR SMALL INTERVALS}

Let $x_{t}$ be an integrated process. In this section, we will show that $R_{n}(y)$ and $S_{n}(y)$ converge in distribution under regularity conditions. To show this, we first establish that all positive integer moments $E\left(R_{n}(y)\right)^{p}$ and $E\left(S_{n}(y)\right)^{p}$ converge. In the lemma below and everywhere in this paper, $F_{t}(\cdot)$ and $f_{t}(\cdot)$ denote the distribution function and density function of $t^{-1 / 2} x_{t}$, respectively (and therefore, the existence of $f_{t}(\cdot)$ is assumed for all $t$ ). Let $\phi(\cdot)$ denote the density function of the standard normal distribution. For the results of this paper, we need the following assumption:

Assumption 1.. $\Delta x_{t}$ is i.i.d., $\sup _{x \in \mathbb{R}}\left|f_{n}(x)-\phi(x)\right| \rightarrow 0$, and $\sup _{t \geq 1, x \in \mathbb{R}}\left|f_{t}^{\prime}(x)\right|$
$<\infty$.

Note that the condition $\sup _{x \in \mathbb{R}}\left|f_{n}(x)-\phi(x)\right| \rightarrow 0$ implies that a variance rescaling to 1 has been imposed. 
Akonom (1993) considers the case where $\Delta x_{t}$ has characteristic function $\psi(r)$ and is i.i.d., $x_{0}=0, E \Delta x_{t}=0, E\left(\Delta x_{t}\right)^{2}<\infty$. From the arguments in Akonom (1993, p. 61-62), it follows that Assumption 1 is then implied by $E\left(\Delta x_{t}\right)^{2}=1$ and $\int_{-\infty}^{\infty}|r||\psi(r)| d r<\infty$. Akonom (1993) also shows that if for some $\beta>0$, $\lim _{r \rightarrow \infty}|r|^{\beta}|\psi(r)|=0$, then there exists an integer $t^{*}$ such that $\sup _{t \geq t^{*}, x \in \mathbb{R}}\left|f_{t}^{\prime}(x)\right|<$ $\infty$. For this paper, we use Assumption 1, which avoids having to split up a number of summations in the proofs into the $t<t^{*}$ and $t \geq t^{*}$ cases, but there does not seem to be a fundamental difficulty with this generalization. Pötscher (2013) used boundedness of density conditions for $t \geq t^{*}$ for some $t^{*}$.

Let $\Gamma(\cdot)$ denote the gamma function and let $\Delta^{k} \mu_{p}$ denote the $k$ th difference of $\mu_{p}$. We can show the convergence of all positive integer-valued moments of $R_{n}(y)$ and $S_{n}(y)$ and deduce convergence in distribution from that, giving the following result:

\section{LEMMA 1. Under Assumption 1,}

1. for all $y \in \mathbb{R}$, there exists a random variable $R(y)$ with moments $\mu_{p}$ satisfying $\mu_{1}=y \sqrt{2 / \pi}$ and, for $p \geq 2$,

$$
\Delta^{p-1} \mu_{p}=p ! y^{p} 2^{-p / 2} / \Gamma(p / 2+1),
$$

such that

$$
R_{n}(y) \stackrel{d}{\longrightarrow} R(y)
$$

2. for all $y \in \mathbb{R}$, there exists a random variable $S(y)$ with moments $v_{p}$ satisfying $v_{1}=2 y \sqrt{2 / \pi}$ and, for $p \geq 2$,

$$
\Delta^{p-1} v_{p}=p ! y^{p} 2^{p / 2} / \Gamma(p / 2+1),
$$

such that

$$
S_{n}(y) \stackrel{d}{\longrightarrow} S(y) .
$$

Note that the distributions of $R(y)$ and $S(y)$ do not depend on the distribution of $\Delta x_{t}$.

One might incorrectly conjecture, based on the standard FCLT plus continuous mapping theorem reasoning and the occupation times formula, that

$$
\begin{aligned}
R_{n}(y) & =\sum_{t=1}^{n} I\left(0<x_{t} \leq y n^{-1 / 2}\right) \stackrel{d}{\approx} n \int_{0}^{1} I\left(0<W(r) \leq y n^{-1}\right) d r \\
& =n \int_{-\infty}^{\infty} I\left(0<s \leq y n^{-1}\right) L(1, s) d s \stackrel{d}{\approx} y L(1,0) \stackrel{d}{=} y|Z|,
\end{aligned}
$$


where $\stackrel{d}{\approx}$ denotes "having a roughly similar distribution as $n \rightarrow \infty$," $L(t, s)$ denotes Brownian local time, and $Z \sim N(0,1)$. Note that the equivalence $L(1,0) \stackrel{d}{=}|Z|$ follows from Akonom (1993, p. 58). If that were the case, then the second moment of $R(y)$ would have to be $E(y Z)^{2}=y^{2}$. However, by Equation (8), we find

$\mu_{2}=\mu_{1}+y^{2}=y \sqrt{2 / \pi}+y^{2}$

and therefore this conjecture is false and $R(y)$ is not distributed as $y|Z|$.

Using the previous lemma, convergence results for $Y_{n 1}=n^{1 / 2} \min _{\left\{t: 1 \leq t \leq n, x_{t}>0\right\}} x_{t}$ and $Z_{n 1}=n^{1 / 2} \min _{1 \leq t \leq n}\left|x_{t}\right|$ now can be proven:

\section{THEOREM 1. Under Assumption 1,}

1. $\lim _{n \rightarrow \infty} P\left(Y_{n 1} \leq y\right)$ is well-defined for all $y \in \mathbb{R}$, and the limit is Lipschitz continuous for $y \in \mathbb{R}$. Furthermore, $Y_{n 1}^{-1}$ converges in distribution.

2. $\lim _{n \rightarrow \infty} P\left(Z_{n 1} \leq y\right)$ is well-defined for all $y \in \mathbb{R}$, and the limit is Lipschitz. continuous for $y \in \mathbb{R}$. Furthermore, $Z_{n 1}^{-1}$ converges in distribution.

Since the limit results of Theorem 1 are based on the observation of Equations (5) and (7), the limits found in Theorem 1 do not depend on the distribution of $\Delta x_{t}$, because the distributions of $R(y)$ and $S(y)$ do not depend on the distribution of $\Delta x_{t}$.

Theorem 1 does not rule out the possibility that some of the probability mass of $Y_{n 1}$ or $Z_{n 1}$ escapes to infinity asymptotically. Therefore, the limit measures of $Y_{n 1}$ and $Z_{n 1}$ are not necessarily probability measures. This is the reason that Theorem 1 is not formulated as a convergence in distribution result. However, Theorem 1 implies that any continuous and bounded function of $Y_{n 1}$ or $Z_{n 1}$ converges in distribution.

Chung (2001, p. 85) uses the term "vague convergence" for the concept of convergence of a sequence of probability measures to a limit measure that is not necessarily a probability measure. Therefore, the result of Theorem 1 implies that $Y_{n 1}$ and $Z_{n 1}$ converge vaguely in Chung's sense.

Note that not all probability mass of $Y_{n 1}$ and $Z_{n 1}$ escapes to infinity. After all, if all probability mass of $Y_{n 1}$ escaped to infinity, we would have $\lim _{n \rightarrow \infty} P\left(Y_{n 1} \leq y\right)=0$ for all $y>0$, implying that for all $y>0$, by Equation (7) and Lemma 1 ,

$0=\lim _{n \rightarrow \infty} P\left(Y_{n 1} \leq y\right)=P(R(y)>1 / 2)=0$,

and because $R(y) \in \mathbb{N}$, this would imply that $R(y)=0$ almost surely, which contradicts our earlier finding that $\mu_{1}>0$ for $y>0$. The same argument holds for $Z_{n 1}$. 


\section{JOINT CONVERGENCE OF OCCUPATION TIMES FOR SMALL INTERVALS}

In this section, we derive multivariate equivalents to the results from the previous section. Consider $\left(Y_{n 1}, Y_{n 2}\right)^{\prime}$, where $Y_{n 1}$ is as before and $Y_{n 2}$ is $n^{1 / 2}$ times the second smallest positive value for $x_{t}$. Assume that $n$ is large enough for $\left\{x_{t}: x_{t}>0, t=\right.$ $1, \ldots, n\}$ to have at least two elements, so that $\left(Y_{n 1}, Y_{n 2}\right)^{\prime}$ are well-defined. The joint distribution of $\left(Y_{n 1}, Y_{n 2}\right)^{\prime}$ then satisfies, for all $y_{1}, y_{2} \in \mathbb{R}$,

$P\left(Y_{n 1} \leq y_{1}, Y_{n 2} \leq y_{2}\right)=P\left(R_{n}\left(y_{1}\right)>1 / 2, R_{n}\left(y_{2}\right)>3 / 2\right)$.

Analogously, assuming that $n$ is large enough for $\left\{x_{t}: x_{t}>0, t=1, \ldots, n\right\}$ to have at least $m$ elements, defining $Y_{n i}$ as the $i$ th smallest positive value for $n^{1 / 2} x_{t}$ for $i=1, \ldots, m$, for all $y_{1}, y_{2}, \ldots, y_{m} \in \mathbb{R}$

$P\left(Y_{n 1} \leq y_{1}, \ldots, Y_{n m} \leq y_{m}\right)=P\left(R_{n}\left(y_{i}\right)>i-1 / 2 \quad \forall i \in\{1, \ldots, m\}\right)$.

A similar observation can be made for $Z_{n i}$, which is defined as the $i$ th smallest value of $n^{1 / 2}\left|x_{t}\right|$. With these definitions and observations in place, we can now find the following joint convergence result for the occupation times of $Y_{n i}$ and $Z_{n i}$ :

\section{LEMMA 2. Under Assumption 1,}

1. for all $\left(y_{1}, y_{2}, \ldots, y_{m}\right)^{\prime} \in \mathbb{R}^{m}$ there exists a random variable $\left(R\left(y_{1}\right), R\left(y_{2}\right), \ldots\right.$, $\left.R\left(y_{m}\right)\right)^{\prime}$ such that

$$
\left(R_{n}\left(y_{1}\right), R_{n}\left(y_{2}\right), \ldots, R_{n}\left(y_{m}\right)\right)^{\prime} \stackrel{d}{\longrightarrow}\left(R\left(y_{1}\right), R\left(y_{2}\right), \ldots, R\left(y_{m}\right)\right)^{\prime} ;
$$

2. for all $\left(y_{1}, y_{2}, \ldots, y_{m}\right)^{\prime} \in \mathbb{R}^{m}$ there exists a random variable $\left(S\left(y_{1}\right), S\left(y_{2}\right), \ldots\right.$, $\left.S\left(y_{m}\right)\right)^{\prime}$ such that

$$
\left(S_{n}\left(y_{1}\right), S_{n}\left(y_{2}\right), \ldots, S_{n}\left(y_{m}\right)\right)^{\prime} \stackrel{d}{\longrightarrow}\left(S\left(y_{1}\right), S\left(y_{2}\right), \ldots, S\left(y_{m}\right)\right)^{\prime} .
$$

Inspecting the proof of Lemma 2 again reveals that the distributions of $\left(R\left(y_{1}\right), R\left(y_{2}\right), \ldots, R\left(y_{m}\right)\right)^{\prime}$ and $\left(S\left(y_{1}\right), S\left(y_{2}\right), \ldots, S\left(y_{m}\right)\right)^{\prime}$ do not depend on the distribution of $\Delta x_{t}$.

Our multivariate results for $\left(Y_{n 1}, \ldots, Y_{n m}\right)^{\prime}$ and $\left(Z_{n 1}, \ldots, Z_{n m}\right)^{\prime}$ are as follows:

THEOREM 2. Under Assumption 1,

1. $\lim _{n \rightarrow \infty} P\left(Y_{n 1} \leq y_{1}, Y_{n 2} \leq y_{2}, \ldots, Y_{n m} \leq y_{m}\right)$ is well-defined for all $\left(y_{1}, \ldots, y_{m}\right)^{\prime} \in$ $\mathbb{R}^{m}$, and the limit is Lipschitz continuous for $\left(y_{1}, \ldots, y_{m}\right)^{\prime} \in \mathbb{R}^{m}$. Furthermore, $\left(Y_{n 1}^{-1}, Y_{n 2}^{-1}, \ldots, Y_{n m}^{-1}\right)^{\prime}$ converges in distribution.

2. $\lim _{n \rightarrow \infty} P\left(Z_{n 1} \leq y_{1}, Z_{n 2} \leq y_{2}, \ldots, Z_{n m} \leq y_{m}\right)$ is well-defined for all $\left(y_{1}, \ldots, y_{m}\right)^{\prime} \in$ $\mathbb{R}^{m}$, and the limit is Lipschitz continuous for $\left(y_{1}, \ldots, y_{m}\right)^{\prime} \in \mathbb{R}^{m}$. Furthermore, $\left(Z_{n 1}^{-1}, Z_{n 2}^{-1}, \ldots, Z_{n m}^{-1}\right)^{\prime}$ converges in distribution. 
Similarly to Theorem 1, the limits found in Theorem 2 do not depend on the distribution of $\Delta x_{t}$ because they are induced by the limit distributions $\left(R\left(y_{1}\right), R\left(y_{2}\right), \ldots, R\left(y_{m}\right)\right)^{\prime}$ and $\left(S\left(y_{1}\right), S\left(y_{2}\right), \ldots, S\left(y_{m}\right)\right)^{\prime}$.

\section{SUMMATIONS OF NEGATIVE POWERS}

This section considers statistics

$n^{-q / 2} \sum_{t=1}^{n} x_{t}^{-q} I\left(x_{t}>0\right)$

and

$n^{-q / 2} \sum_{t=1}^{n}\left|x_{t}\right|^{-q}$

for $q>1$. These statistics have also been considered by Pötscher (2013); in this section, we will find their limit distributions using Theorem 2 of the previous section. The idea here is that the statistic $n^{-q / 2} \sum_{t=1}^{n} x_{t}^{-q} I\left(x_{t}>0\right)$ can be written as $\sum_{t=1}^{M_{n}} Y_{n t}^{-q}$, where $M_{n}$ is the number of positive $x_{t}$, and that the last statistic is asymptotically close to $\sum_{t=1}^{m} Y_{n t}^{-q}$ for large $m$. By the joint convergence in distribution result of Theorem 2, and letting $\left(Y_{1}^{-1}, \ldots, Y_{m}^{-1}\right)^{\prime}$ denote a random variable that has the limit distribution of $\left(Y_{n 1}^{-1}, \ldots, Y_{n m}^{-1}\right)^{\prime}$, we find that $\sum_{t=1}^{m} Y_{n t}^{-q} \stackrel{d}{\longrightarrow} \sum_{t=1}^{m} Y_{t}^{-q}$, and since $m$ was arbitrary, we find the limit distribution as $\sum_{t=1}^{\infty} Y_{t}^{-q}$. A similar result holds for $n^{-q / 2} \sum_{t=1}^{n}\left|x_{t}\right|^{-q}$, defining $\left(Z_{1}^{-1}, \ldots, Z_{m}^{-1}\right)^{\prime}$ analogously. This argument then leads to the following result:

THEOREM 3. For $q>1$, under Assumption 1,

$n^{-q / 2} \sum_{t=1}^{n} x_{t}^{-q} I\left(x_{t}>0\right) \stackrel{d}{\longrightarrow} \sum_{t=1}^{\infty} Y_{t}^{-q}$

and

$n^{-q / 2} \sum_{t=1}^{n}\left|x_{t}\right|^{-q} \stackrel{d}{\longrightarrow} \sum_{t=1}^{\infty} Z_{t}^{-q}$,

and the limit distributions do not depend on the distribution of the innovations $\Delta x_{t}$.

\section{SIMULATION RESULTS}

We conducted a small simulation experiment to illustrate the main theorems. We simulated $\min _{\left\{t: 1 \leq t \leq n, x_{t}>0\right\}} x_{t}, \min _{1 \leq t \leq n}\left|x_{t}\right|, n^{-1} \sum_{t=1}^{n} x_{t}^{-2} I\left(x_{t}>0\right)$ and $n^{-1}$ $\sum_{t=1}^{n} x_{t}^{-2}$ for various values on $n$ and i.i.d. $N(0,1)$ distributed $\Delta x_{t}$, and reported the percentage points in Tables A1-A4. For all four statistics, the convergence of the distribution was rapid. Note that the "NA" for $n=100$ in Table A1 for the 95th percentage point corresponds to the occurrence in more than $5 \%$ of cases of an integrated process that was always negative. 


\section{APPENDICES}

\section{A. Simulation results}

TABLE A1. Simulation results for $n^{1 / 2} \min _{\left\{t: 1 \leq t \leq n, x_{t}>0\right\}} x_{t}$

\begin{tabular}{lcccccccc}
\hline$n$ & $\begin{array}{c}\text { No. of } \\
\text { replications }\end{array}$ & $5 \%$ & $10 \%$ & $25 \%$ & $50 \%$ & $75 \%$ & $90 \%$ & $95 \%$ \\
\hline 100 & $10^{7}$ & 0.070 & 0.146 & 0.419 & 1.142 & 2.990 & 8.296 & NA \\
1,000 & $10^{6}$ & 0.067 & 0.139 & 0.401 & 1.103 & 2.924 & 7.896 & 16.065 \\
10,000 & $10^{5}$ & 0.067 & 0.138 & 0.396 & 1.095 & 2.884 & 7.766 & 15.664 \\
100,000 & $10^{5}$ & 0.066 & 0.137 & 0.396 & 1.094 & 2.920 & 7.828 & 15.835 \\
\hline
\end{tabular}

TABLE A2. Simulation results for $n^{1 / 2} \min _{1 \leq t \leq n}\left|x_{t}\right|$

\begin{tabular}{lcccccccc}
\hline$n$ & $\begin{array}{c}\text { No. of } \\
\text { replications }\end{array}$ & $5 \%$ & $10 \%$ & $25 \%$ & $50 \%$ & $75 \%$ & $90 \%$ & $95 \%$ \\
\hline 100 & $10^{7}$ & 0.035 & 0.073 & 0.209 & 0.571 & 1.489 & 3.888 & 7.122 \\
1,000 & $10^{6}$ & 0.033 & 0.070 & 0.201 & 0.553 & 1.460 & 3.943 & 7.919 \\
10,000 & $10^{5}$ & 0.034 & 0.070 & 0.199 & 0.550 & 1.460 & 3.917 & 7.854 \\
100,000 & $10^{5}$ & 0.033 & 0.069 & 0.199 & 0.550 & 1.462 & 3.962 & 7.922 \\
\hline
\end{tabular}

TABLE A3. Simulation results for $n^{-1} \sum_{t=1}^{n} x_{t}^{-2} I\left(x_{t}>0\right)$

\begin{tabular}{lcccccccc}
\hline$n$ & $\begin{array}{c}\text { No. of } \\
\text { replications }\end{array}$ & $5 \%$ & $10 \%$ & $25 \%$ & $50 \%$ & $75 \%$ & $90 \%$ & $95 \%$ \\
\hline 100 & $10^{7}$ & 0.000 & 0.045 & 0.256 & 1.399 & 7.958 & 54.230 & 219.790 \\
1,000 & $10^{6}$ & 0.012 & 0.041 & 0.267 & 1.517 & 8.767 & 59.559 & 242.223 \\
10,000 & $10^{5}$ & 0.010 & 0.041 & 0.271 & 1.553 & 9.019 & 61.381 & 243.012 \\
100,000 & $10^{5}$ & 0.010 & 0.040 & 0.265 & 1.559 & 9.001 & 61.915 & 254.565 \\
\hline
\end{tabular}

TABLE A4. Simulation results for $n^{-1} \sum_{t=1}^{n} x_{t}^{-2}$

\begin{tabular}{lcccccccc}
\hline$n$ & $\begin{array}{c}\text { No. of } \\
\text { replications }\end{array}$ & $5 \%$ & $10 \%$ & $25 \%$ & $50 \%$ & $75 \%$ & $90 \%$ & $95 \%$ \\
\hline 100 & $10^{7}$ & 0.072 & 0.184 & 1.029 & 5.632 & 31.939 & 217.331 & 877.243 \\
1,000 & $10^{6}$ & 0.044 & 0.162 & 1.066 & 6.073 & 34.930 & 239.072 & 968.151 \\
10,000 & $10^{5}$ & 0.042 & 0.160 & 1.071 & 6.155 & 35.852 & 237.745 & 939.627 \\
100,000 & $10^{5}$ & 0.039 & 0.155 & 1.068 & 6.218 & 35.930 & 244.387 & 995.949 \\
\hline
\end{tabular}




\section{B. Mathematical proofs}

In this appendix, for brevity we will set $I_{t 1}=I\left(0<x_{t} \leq y n^{-1 / 2}\right)$ and $I_{t 2}=I\left(\left|x_{t}\right| \leq y n^{-1 / 2}\right)$. We will also define $I_{t, y, 1}=I\left(0<x_{t} \leq y n^{-1 / 2}\right)$ and $I_{t, y, 2}=I\left(\left|x_{t}\right| \leq y n^{-1 / 2}\right)$ whenever three instead of two arguments are used for $I$.

\section{Proof of Lemma 1}

The proof of Lemma 1 relies on Lemmas 3-10 below.

LEMMA 3. A random sequence $X_{n} \in \mathbb{R}$ converges in distribution to a random variable $X$ with moments $\zeta_{p}$ if (1) $E X_{n}^{p}$ converges to a limit $\zeta_{p}$ for all $p \in \mathbb{N}$; and (2) $\sum_{p=1}^{\infty} \zeta_{2 p}^{-1 /(2 p)}$ $=\infty$.

Proof of Lemma 3. This result follows from Fréchet and Shohat (1931) as quoted on page 2 of Lin (2017), together with Lin's Theorem 1 and condition (h7).

LEMMA 4. As $n \rightarrow \infty$, for $p \geq 2$,

$$
\begin{aligned}
n^{-p / 2} & \sum_{t_{1}=1}^{n} \ldots \sum_{t_{p}=1}^{n} I\left(t_{1}<t_{2}\right) \ldots I\left(t_{p-1}<t_{p}\right) t_{1}^{-1 / 2}\left(t_{2}-t_{1}\right)^{-1 / 2} \ldots\left(t_{p}-t_{p-1}\right)^{-1 / 2} \\
& \rightarrow \int_{0}^{1} \int_{0}^{s_{p}} \ldots \int_{0}^{s_{2}} s_{1}^{-1 / 2}\left(s_{2}-s_{1}\right)^{-1 / 2} \ldots\left(s_{p-1}-s_{p-2}\right)^{-1 / 2}\left(s_{p}-s_{p-1}\right)^{-1 / 2} d s_{1} \ldots d s_{p} .
\end{aligned}
$$

Proof of Lemma 4. Note that, by letting [.] denote the floor function and setting $t_{1}=j_{1}$ and $t_{i}-t_{i-1}=j_{i}$ for $i=2, \ldots, p$, and then $j_{i}=n x_{i}+1$ for $i=1, \ldots, p$,

$$
\begin{aligned}
n^{-p / 2} & \sum_{t_{1}=1}^{n} \ldots \sum_{t_{p}=1}^{n} I\left(t_{1}<t_{2}\right) \ldots I\left(t_{p-1}<t_{p}\right) t_{1}^{-1 / 2}\left(t_{2}-t_{1}\right)^{-1 / 2} \ldots\left(t_{p}-t_{p-1}\right)^{-1 / 2} \\
= & n^{-p / 2} \sum_{j_{1}=1}^{n} \ldots \sum_{j_{p}=1}^{n} I\left(1 \leq j_{1}+j_{2} \leq n\right) \ldots I\left(1 \leq j_{1}+\cdots+j_{p} \leq n\right) j_{1}^{-1 / 2} j_{2}^{-1 / 2} \ldots j_{p}^{-1 / 2} \\
= & n^{-p / 2} \int_{j_{1}=1}^{n+1} \ldots \int_{j_{p}=1}^{n+1} I\left(1 \leq\left[j_{1}\right]+\left[j_{2}\right] \leq n\right) \ldots \\
& \times I\left(1 \leq\left[j_{1}\right]+\cdots+\left[j_{p}\right] \leq n\right)\left[j_{1}\right]^{-1 / 2}\left[j_{2}\right]^{-1 / 2} \ldots\left[j_{p}\right]^{-1 / 2} d j_{1} \ldots d j_{p} \\
= & n^{p / 2} \int_{x_{1}=0}^{1} \ldots \int_{x_{p}=0}^{1} I\left(1 \leq\left[n x_{1}+1\right]+\left[n x_{2}+1\right] \leq n\right) \ldots \\
& I\left(1 \leq\left[n x_{1}+1\right]+\cdots+\left[n x_{p}+1\right] \leq n\right) \\
& \times\left[n x_{1}+1\right]^{-1 / 2}\left[n x_{2}+1\right]^{-1 / 2} \ldots\left[n x_{p}+1\right]^{-1 / 2} d x_{1} \ldots d x_{p} .
\end{aligned}
$$

Pointwise for $\left(x_{1}, \ldots, x_{p}\right) \in(0,1]^{p}$,

$$
\begin{aligned}
& n^{p / 2} I\left(1 \leq\left[n x_{1}+1\right]+\left[n x_{2}+1\right] \leq n\right) \ldots I\left(1 \leq\left[n x_{1}+1\right]+\cdots+\left[n x_{p}+1\right] \leq n\right) \\
& \quad \times\left[n x_{1}+1\right]^{-1 / 2}\left[n x_{2}+1\right]^{-1 / 2} \ldots\left[n x_{p}+1\right]^{-1 / 2}
\end{aligned}
$$




$$
\rightarrow I\left(x_{1}+x_{2} \leq 1\right) \ldots I\left(x_{1}+\cdots+x_{p} \leq 1\right) x_{1}^{-1 / 2} x_{2}^{-1 / 2} \ldots x_{p}^{-1 / 2}
$$

and therefore, by the dominated convergence theorem, it suffices to find an integrable dominating function. To find the dominating function, note that because $[x+1] \geq x$

$$
\begin{array}{rl}
n^{p / 2} & I\left(1 \leq\left[n x_{1}+1\right]+\left[n x_{2}+1\right] \leq n\right) \ldots I\left(1 \leq\left[n x_{1}+1\right]+\cdots+\left[n x_{p}+1\right] \leq n\right) \\
& \times\left[n x_{1}+1\right]^{-1 / 2}\left[n x_{2}+1\right]^{-1 / 2} \ldots\left[n x_{p}+1\right]^{-1 / 2} \\
\leq & x_{1}^{-1 / 2} x_{2}^{-1 / 2} \ldots x_{p}^{-1 / 2}
\end{array}
$$

which is integrable. Therefore, the limit of the statistic of Equation (23) is

$$
\begin{aligned}
& \int_{0}^{1} \ldots \int_{0}^{1} I\left(x_{1}+x_{2} \leq 1\right) \ldots I\left(x_{1}+\cdots+x_{p} \leq 1\right) x_{1}^{-1 / 2} x_{2}^{-1 / 2} \ldots x_{p}^{-1 / 2} d x_{1} \ldots d x_{p} \\
& =\int_{-\infty}^{\infty} \ldots \int_{-\infty}^{\infty} I\left(0 \leq x_{1} \leq 1\right) \ldots I\left(0 \leq x_{p} \leq 1\right) I\left(x_{1}+x_{2} \leq 1\right) \ldots I\left(x_{1}+\cdots+x_{p} \leq 1\right) \\
& \quad \times x_{1}^{-1 / 2} x_{2}^{-1 / 2} \ldots x_{p}^{-1 / 2} d x_{1} \ldots d x_{p} .
\end{aligned}
$$

Now set $x_{1}=s_{1}, x_{1}+x_{2}=s_{2}, x_{1}+x_{2}+x_{3}=s_{3}$, etc. Then the last expression can be rewritten as

$$
\begin{aligned}
& \int_{0}^{1} \ldots \int_{0}^{1} I\left(0 \leq s_{1} \leq 1\right) I\left(s_{1} \leq s_{2} \leq 1\right) \ldots I\left(s_{p-1} \leq s_{p} \leq 1\right) \\
& \quad \times s_{1}^{-1 / 2}\left(s_{2}-s_{1}\right)^{-1 / 2} \ldots\left(s_{p}-s_{p-1}\right)^{-1 / 2} d s_{1} \ldots d s_{p} \\
& =\int_{0}^{1} \int_{0}^{s_{p}} \ldots \int_{0}^{s_{2}} s_{1}^{-1 / 2}\left(s_{2}-s_{1}\right)^{-1 / 2} \ldots\left(s_{p-1}-s_{p-2}\right)^{-1 / 2}\left(s_{p}-s_{p-1}\right)^{-1 / 2} d s_{1} \ldots d s_{p},
\end{aligned}
$$

which is the result as stated in the lemma.

LEMMA 5. For $p \geq 1$, setting $s_{0}=0$,

$$
\begin{aligned}
\int_{s_{p}=}^{s_{p}=1} & \int_{s_{p-1}=0}^{s_{p}} \ldots \int_{s_{1}=0}^{s_{1}=s_{2}}\left(s_{p}-s_{p-1}\right)^{-1 / 2}\left(s_{p-1}-s_{p-2}\right)^{-1 / 2} \ldots\left(s_{2}-s_{1}\right)^{-1 / 2} s_{1}^{-1 / 2} \\
& \times d s_{1} \ldots d s_{p} \\
= & (\Gamma(1 / 2))^{p} / \Gamma(p / 2+1) .
\end{aligned}
$$

Proof of Lemma 5. Equations (2.2) and (2.3) on p. 44 and 45 of Miller and Ross (1993) state the following two properties of the Riemann-Liouville fractional integral:

(R1) For $\alpha>0, D_{t}^{-\alpha} f(t)=(\Gamma(\alpha))^{-1} \int_{0}^{t}(t-\tau)^{\alpha-1} f(\tau) d \tau$.

(R2) For $\alpha>0$ and $\beta>-1, D_{t}^{-\alpha} t^{\beta}=\Gamma(\beta+1) t^{\alpha+\beta} / \Gamma(\alpha+\beta+1)$. 
For $p=1$, we use (R1) and (R2) using $\alpha=1, \beta=-1 / 2$, and $f(\tau)=\tau^{-1 / 2}$, find that

$\int_{0}^{1} s_{1}^{-1 / 2} d s_{1}=\left.\Gamma(1) D_{t}^{-1} t^{-1 / 2}\right|_{t=1}=\Gamma(1 / 2) / \Gamma(3 / 2)=2$.

For $p=2$, we have

$$
\begin{aligned}
\int_{0}^{1} \int_{0}^{s_{2}}\left(s_{2}-s_{1}\right)^{-1 / 2} s_{1}^{-1 / 2} d s_{1} d s_{2} & =\int_{0}^{1} \Gamma(1 / 2) D_{s_{2}}^{-1 / 2} s_{2}^{-1 / 2} d s_{2} \\
& =\left.\Gamma(1 / 2) D_{t}^{-1} D_{t}^{-1 / 2} t^{-1 / 2}\right|_{t=1},
\end{aligned}
$$

by applying (R1) twice (with $\alpha=1 / 2, t=s_{2}, \tau=s_{1}, f(\tau)=\tau^{-1 / 2}$ and with $\alpha=1, \tau=s_{2}$, $f(\tau)=D_{\tau}^{-1 / 2} \tau^{-1 / 2}$ ). Setting $\alpha=3 / 2$ and $\beta=-1 / 2$, we apply (R2) to the above expression and find that it equals

$\Gamma(1 / 2) \Gamma(1 / 2) t /\left.\Gamma(2)\right|_{t=1}=(\Gamma(1 / 2))^{2} / \Gamma(2)=\pi$.

For $p=3$, note that

$$
\begin{gathered}
\int_{0}^{1} \int_{0}^{s_{3}}\left(s_{3}-s_{2}\right)^{-1 / 2} \int_{0}^{s_{2}}\left(s_{2}-s_{1}\right)^{-1 / 2} s_{1}^{-1 / 2} d s_{1} d s_{2} d s_{3} \\
=\int_{0}^{1} \int_{0}^{s_{3}}\left(s_{3}-s_{2}\right)^{-1 / 2} \Gamma(1 / 2) D_{s_{2}}^{-1 / 2} s_{2}^{-1 / 2} d s_{2} d s_{3} .
\end{gathered}
$$

Applying (R1) twice (with $\alpha=1 / 2, t=s_{3}, \tau=s_{2}, f(\tau)=D_{\tau}^{-1 / 2} \tau^{-1 / 2}$ and with $\alpha=1$, $\left.\tau=s_{3}, f(\tau)=D_{\tau}^{-1} \tau^{-1 / 2}\right)$ gives

$$
\begin{aligned}
\int_{0}^{1} & \int_{0}^{s_{3}}\left(s_{3}-s_{2}\right)^{-1 / 2} \Gamma(1 / 2) D_{s_{2}}^{-1 / 2} s_{2}^{-1 / 2} d s_{2} d s_{3} \\
\quad= & \int_{0}^{1}(\Gamma(1 / 2))^{2} D_{s_{3}}^{-1} s_{3}^{-1 / 2} d s_{3}=\left.(\Gamma(1 / 2))^{2} D_{t}^{-2} t^{-1 / 2}\right|_{t=1} .
\end{aligned}
$$

By setting $\alpha=2$ and $\beta=-1 / 2$, (R2) implies that the expression is equal to $(\Gamma(1 / 2))^{2} \Gamma(1 / 2) t^{3 / 2} /\left.\Gamma(5 / 2)\right|_{t=1}=(\Gamma(1 / 2))^{3} / \Gamma(5 / 2)=4 \pi / 3$.

Reasoning accordingly, we now find that the $p$-fold integral is $\int_{0}^{1}(\Gamma(1 / 2))^{p-1} D_{s_{p}}^{-p / 2+1 / 2} s_{p}^{-1 / 2} d s_{p}=\left.D_{t}^{-1}(\Gamma(1 / 2))^{p-1} D_{t}^{-p / 2+1 / 2} t^{-1 / 2}\right|_{t=1}$,

by (R1). By setting $\alpha=p / 2+1 / 2$ and $\beta=-1 / 2$, (R2) implies that the above expression is equal to

$(\Gamma(1 / 2))^{p-1} \Gamma(1 / 2) t^{p / 2} /\left.\Gamma(p / 2+1)\right|_{t=1}=(\Gamma(1 / 2))^{p} / \Gamma(p / 2+1)$,

thereby completing the proof.

LEMMA 6. Assume Assumption 1 holds. Then we have $\lim _{n \rightarrow \infty} E\left(\sum_{t=1}^{n} I_{t 1}\right)=y \sqrt{2 / \pi}$ 
and

$$
\lim _{n \rightarrow \infty} E\left(\sum_{t=1}^{n} I_{t 2}\right)=2 y \sqrt{2 / \pi}
$$

and for $p \geq 2$,

$$
\begin{aligned}
& \lim _{n \rightarrow \infty} \sum_{t_{1}=1}^{n} \ldots \sum_{t_{p}=1}^{n} E\left(I_{t_{1}, 1} \ldots I_{t_{p}, 1}\right) I\left(t_{1} \neq t_{2}\right) I\left(t_{2} \neq t_{3}\right) \ldots I\left(t_{p-1} \neq t_{p}\right) \\
& \quad=p ! y^{p} 2^{-p / 2} / \Gamma(p / 2+1)
\end{aligned}
$$

and

$$
\begin{aligned}
& \lim _{n \rightarrow \infty} \sum_{t_{1}=1}^{n} \ldots \sum_{t_{p}=1}^{n} E\left(I_{t_{1}, 2} \ldots I_{t_{p}, 2}\right) I\left(t_{1} \neq t_{2}\right) I\left(t_{2} \neq t_{3}\right) \ldots I\left(t_{p-1} \neq t_{p}\right) \\
& \quad=p ! y^{p} 2^{p / 2} / \Gamma(p / 2+1) .
\end{aligned}
$$

Proof of Lemma 6. We will first show the first assertion of the lemma. By the Taylor expansion, for some intermediate value $\xi_{\text {tny }} \in\left[0, y t^{-1 / 2} n^{-1 / 2}\right]$,

$$
\begin{aligned}
\mu_{1} & =\lim _{n \rightarrow \infty} E\left(\sum_{t=1}^{n} I_{t 1}\right)=\lim _{n \rightarrow \infty} \sum_{t=1}^{n}\left(F_{t}\left(y t^{-1 / 2} n^{-1 / 2}\right)-F_{t}(0)\right) \\
& =\lim _{n \rightarrow \infty} y n^{-1 / 2} \sum_{t=1}^{n} t^{-1 / 2} f_{t}\left(\xi_{\text {tny }}\right) \\
& =2 y \phi(0)=y \sqrt{2 / \pi}
\end{aligned}
$$

because $n^{-1 / 2} \sum_{t=1}^{n} t^{-1 / 2} \rightarrow 2$ and because $\sup _{x \in \mathbb{R}}\left|f_{t}^{\prime}(x)\right|<\infty$ and $\sup _{t \geq 1, x \in \mathbb{R}} \mid f_{t}(x)-$ $\phi(x) \mid \rightarrow 0$ by assumption. The result for $\lim _{n \rightarrow \infty} E\left(\sum_{t=1}^{n} I_{t 2}\right)$ follows analogously.

To show the second assertion of the lemma, note that since there are $p$ ! possible orderings of $\left\{t_{1}, t_{2}, \ldots, t_{p}\right\}$,

$$
\begin{aligned}
& \sum_{t_{1}=1}^{n} \ldots \sum_{t_{p}=1}^{n} E\left(I_{t_{1}, 1} \ldots I_{t_{p}, 1}\right) I\left(t_{1} \neq t_{2}\right) I\left(t_{2} \neq t_{3}\right) \ldots I\left(t_{p-1} \neq t_{p}\right) \\
& \quad=p ! \sum_{t_{1}=1}^{n} \ldots \sum_{t_{p}=1}^{n} E\left(I_{t_{1}, 1} \ldots I_{t_{p}, 1}\right) I\left(t_{1}<t_{2}\right) I\left(t_{2}<t_{3}\right) \ldots I\left(t_{p-1}<t_{p}\right) .
\end{aligned}
$$

Letting $g_{t}(\cdot)$ denote the density of $x_{t}$, we have

$$
\begin{aligned}
& E\left(I_{t_{1}, 1} \ldots I_{t_{p}, 1}\right)=E\left(I\left(0 \leq x_{t_{1}} \leq y n^{-1 / 2}\right) \ldots I\left(0 \leq x_{t_{p}} \leq y n^{-1 / 2}\right)\right) \\
& =E\left(I\left(0 \leq x_{t_{p}}-x_{t_{p-1}}+\cdots+x_{t_{2}}-x_{t_{1}}+x_{t_{1}} \leq y n^{-1 / 2}\right) \ldots I\left(0 \leq x_{t_{1}} \leq y n^{-1 / 2}\right)\right) \\
& =\int \ldots \int I\left(0 \leq z_{1}+z_{2}+\cdots+z_{p} \leq y n^{-1 / 2}\right) \ldots I\left(0 \leq z_{1} \leq y n^{-1 / 2}\right) \\
& \quad \times g_{t_{1}}\left(z_{1}\right) g_{t_{2}-t_{1}}\left(z_{2}\right) \ldots g_{t_{p}-t_{p-1}}\left(z_{p}\right) d z_{1} \ldots d z_{p} .
\end{aligned}
$$


Since $g_{t}(z)=t^{-1 / 2} f_{t}\left(t^{-1 / 2} z\right), \sup _{|z| \leq y n^{-1 / 2}}\left|g_{t}(z)-t^{-1 / 2} \phi(0)\right|=o\left(t^{-1 / 2}\right)$ because

$$
\begin{aligned}
c_{t} & =\sup _{|z| \leq y n^{-1 / 2}}\left|g_{t}(z)-t^{-1 / 2} \phi(0)\right|=\sup _{|z| \leq y n^{-1 / 2}}\left|t^{-1 / 2} f_{t}\left(t^{-1 / 2} z\right)-t^{-1 / 2} \phi(0)\right| \\
& \leq t^{-1 / 2} \sup _{|z| \leq y n^{-1 / 2}}\left(\left|f_{t}\left(t^{-1 / 2} z\right)-f_{t}(0)\right|+\left|f_{t}(0)-\phi(0)\right|\right)=o\left(t^{-1 / 2}\right)
\end{aligned}
$$

because by assumption $\sup _{x \in \mathbb{R}}\left|f_{t}(x)-\phi(x)\right| \rightarrow 0$ and $\sup _{t \geq 1, x \in \mathbb{R}}\left|f_{t}^{\prime}(x)\right|<\infty$. Therefore, approximating $g_{t_{1}}(z)$ by $t^{-1 / 2} \phi(0)$ gives

$$
\begin{aligned}
& \mid \sum_{t_{1}=1}^{n} \ldots \sum_{t_{p}=1}^{n} I\left(t_{1}<t_{2}\right) \ldots I\left(t_{p-1}<t_{p}\right) E\left(I_{t_{1}, 1} \ldots I_{t_{p}, 1}\right) \\
& -\sum_{t_{1}=1}^{n} \ldots \sum_{t_{p}=1}^{n} I\left(t_{1}<t_{2}\right) \ldots I\left(t_{p-1}<t_{p}\right) \\
& \times \int \ldots \int I\left(0 \leq z_{1}+z_{2}+\cdots+z_{p} \leq y n^{-1 / 2}\right) \ldots I\left(0 \leq z_{1} \leq y n^{-1 / 2}\right) \\
& \times t_{1}^{-1 / 2} \phi(0) g_{t_{2}-t_{1}}\left(z_{2}\right) \ldots g_{t_{p}-t_{p-1}}\left(z_{p}\right) d z_{1} \ldots d z_{p} \mid \\
& \leq \sum_{t_{1}=1}^{n} c_{t_{1}} \sum_{t_{2}=1}^{n} \ldots \sum_{t_{p}=1}^{n} I\left(t_{1}<t_{2}\right) \ldots I\left(t_{p-1}<t_{p}\right) \\
& \times \int \ldots \int I\left(0 \leq z_{1}+z_{2}+\cdots+z_{p} \leq y n^{-1 / 2}\right) \ldots I\left(0 \leq z_{1} \leq y n^{-1 / 2}\right) \\
& \times g_{t_{2}-t_{1}}\left(z_{2}\right) \ldots g_{t_{p}-t_{p-1}}\left(z_{p}\right) d z_{1} \ldots d z_{p} \\
& =\sum_{t_{1}=1}^{n} c_{t_{1}} \sum_{t_{2}=1}^{n} \ldots \sum_{t_{p}=1}^{n} I\left(t_{1}<t_{2}\right) \ldots I\left(t_{p-1}<t_{p}\right)\left(t_{2}-t_{1}\right)^{-1 / 2} \ldots\left(t_{p}-t_{p-1}\right)^{-1 / 2} \\
& \times \int \ldots \int I\left(0 \leq z_{1}+z_{2}+\cdots+z_{p} \leq y n^{-1 / 2}\right) \ldots I\left(0 \leq z_{1} \leq y n^{-1 / 2}\right) \\
& \times f_{t_{2}-t_{1}}\left(z_{2}\right) \ldots f_{t_{p}-t_{p-1}}\left(z_{p}\right) d z_{1} \ldots d z_{p} \\
& \leq \sum_{t_{1}=1}^{n} c_{t_{1}} \sum_{t_{2}=1}^{n}\left(t_{2}-t_{1}\right)^{-1 / 2} \ldots \sum_{t_{p}=1}^{n}\left(t_{p}-t_{p-1}\right)^{-1 / 2} I\left(t_{1}<t_{2}\right) \ldots I\left(t_{p-1}<t_{p}\right)\left(y n^{-1 / 2}\right)^{p} \\
& \times\left(\sup _{t \geq 1, x \in \mathbb{R}} f_{t}(x)\right)^{p-1} \\
& =O\left(n^{-1 / 2} \sum_{t=1}^{n} c_{t}\right)=o(1) \text {. }
\end{aligned}
$$


Similarly, we can also approximate $g_{t_{2}-t_{1}}(z)$ by $\left(t_{2}-t_{1}\right)^{-1 / 2} \phi(0), g_{t_{3}-t_{2}}(z)$ by $\left(t_{3}-\right.$ $\left.t_{2}\right)^{-1 / 2} \phi(0)$, etc. and therefore

$$
\begin{aligned}
\sum_{t_{1}=1}^{n} & \ldots \sum_{t_{p}=1}^{n} I\left(t_{1}<t_{2}\right) \ldots I\left(t_{p-1}<t_{p}\right) E\left(I_{t_{1}, 1} \ldots I_{t_{p}, 1}\right) \\
= & \sum_{t_{1}=1}^{n} \ldots \sum_{t_{p}=1}^{n} I\left(t_{1}<t_{2}\right) \ldots \\
& \times I\left(t_{p-1}<t_{p}\right) \int \ldots \int I\left(0 \leq z_{1}+z_{2}+\cdots+z_{p} \leq y n^{-1 / 2}\right) \ldots I\left(0 \leq z_{1} \leq y n^{-1 / 2}\right) \\
& \times t_{1}^{-1 / 2} \phi(0)^{p}\left(t_{2}-t_{1}\right)^{-1 / 2} \ldots\left(t_{p}-t_{p-1}\right)^{-1 / 2} d z_{1} \ldots d z_{p}+o(1) .
\end{aligned}
$$

Because

$$
\int \ldots \int I\left(0 \leq z_{1}+z_{2}+\cdots+z_{p} \leq y n^{-1 / 2}\right) \ldots I\left(0 \leq z_{1} \leq y n^{-1 / 2}\right) d z_{1} \ldots d z_{p}=y^{p} n^{-p / 2},
$$

it now follows that

$$
\begin{aligned}
\sum_{t_{1}=1}^{n} & \ldots \sum_{t_{p}=1}^{n} I\left(t_{1}<t_{2}\right) \ldots I\left(t_{p-1}<t_{p}\right) E\left(I_{t_{1}, 1} \ldots I_{t_{p}, 1}\right) \\
= & o(1)+\left(y n^{-1 / 2} \phi(0)\right)^{p} \sum_{t_{1}=1}^{n} \ldots \sum_{t_{p}=1}^{n} I\left(t_{1}<t_{2}\right) \ldots I\left(t_{p-1}<t_{p}\right) t_{1}^{-1 / 2}\left(t_{2}-t_{1}\right)^{-1 / 2} \ldots \\
& \times\left(t_{p}-t_{p-1}\right)^{-1 / 2}
\end{aligned}
$$

and by Lemma 4, the last expression equals

$$
\begin{aligned}
& o(1)+y^{p} \phi(0)^{p} \int_{0}^{1}\left(s_{p}-s_{p-1}\right)^{-1 / 2} \int_{0}^{s_{p-1}}\left(s_{p-1}-s_{p-2}\right)^{-1 / 2} \ldots \\
& \quad \times \int_{0}^{s_{2}} s_{1}^{-1 / 2}\left(s_{2}-s_{1}\right)^{-1 / 2} d s_{1} \ldots d s_{p}
\end{aligned}
$$

and by Lemma 5, it now follows that

$$
\begin{aligned}
& \lim _{n \rightarrow \infty} \sum_{t_{1}=1}^{n} \ldots \sum_{t_{p}=1}^{n} E\left(I_{t_{1}, 1} \ldots I_{t_{p}, 1}\right) I\left(t_{1} \neq t_{2}\right) I\left(t_{2} \neq t_{3}\right) \ldots I\left(t_{p-1} \neq t_{p}\right) \\
& =p ! y^{p} \phi(0)^{p}(\Gamma(1 / 2))^{p} / \Gamma(p / 2+1) \\
& =p ! y^{p} 2^{-p / 2} / \Gamma(p / 2+1) .
\end{aligned}
$$

The result for $I_{t 2}$ follows analogously, except that in the result of Equation (24), $y^{p} n^{-p / 2}$ needs to be replaced by $2^{p} y^{p} n^{-p / 2}$. 
LEMMA 7. Assume Assumption 1 holds. For $b=1,2, p \geq 2$ and $j=1,2, \ldots, p-1$, define $h_{b n p j}=\sum_{t_{1}=1}^{n} \sum_{t_{2}=1}^{n} \ldots \sum_{t_{p}=1}^{n} E\left(I_{t_{1}, b} I_{t_{2}, b} \ldots I_{t_{p}, b}\right) I\left(t_{1} \neq t_{2}\right) I\left(t_{2} \neq t_{3}\right) \ldots I\left(t_{j} \neq t_{j+1}\right)$

and for $b=1,2$ and $p \geq 1$, define $h_{b n p 0}=E\left(\sum_{t=1}^{n} I_{t b}\right)^{p}$. Then $H_{b p j}=\lim _{n \rightarrow \infty} h_{b n p j}$ is welldefined for $b=1,2, p \geq 1$ and $j=0,1,2, \ldots, p-1$, and as a consequence, $\mu_{p}=H_{1 p 0}=$ $\lim _{n \rightarrow \infty} h_{1 n p 0}$ and $v_{p}=H_{2 p 0}=\lim _{n \rightarrow \infty} h_{2 n p 0}$ are well-defined for $p \geq 1$. Also, for $b=$ $1,2, p \geq 2$ and $j=0, \ldots, p-2$,

$H_{b p j}=H_{b, p-1, j}+H_{b, p, j+1}$.

Proof of Lemma 7. For $b=1,2, p \geq 3$ and $j=1,2, \ldots, p-2$, we can write

$$
\begin{aligned}
h_{b n p j}= & \sum_{t_{1}=1}^{n} \sum_{t_{2}=1}^{n} \ldots \sum_{t_{p}=1}^{n} E\left(I_{t_{1}, b} I_{t_{2}, b} \ldots I_{t_{p}, b}\right) I\left(t_{1} \neq t_{2}\right) \ldots I\left(t_{j} \neq t_{j+1}\right) \\
= & \sum_{t_{1}=1}^{n} \sum_{t_{2}=1}^{n} \ldots \sum_{t_{p}=1}^{n} E\left(I_{t_{1}, b} I_{t_{2}, b} \ldots I_{t_{p}, b}\right) I\left(t_{1} \neq t_{2}\right) \ldots I\left(t_{j} \neq t_{j+1}\right) \\
& \times\left(I\left(t_{j+1}=t_{j+2}\right)+I\left(t_{j+1} \neq t_{j+2}\right)\right) \\
= & \sum_{t_{1}=1}^{n} \sum_{t_{2}=1}^{n} \ldots \sum_{t_{p-1}=1}^{n} E\left(I_{t_{1}, b} I_{t_{2}, b} \ldots I_{t_{p-1}, b}\right) I\left(t_{1} \neq t_{2}\right) \ldots I\left(t_{j} \neq t_{j+1}\right) \\
& +\sum_{t_{1}=1}^{n} \sum_{t_{2}=1}^{n} \ldots \sum_{t_{p}=1}^{n} E\left(I_{t_{1}, b} I_{t_{2}, b} \ldots I_{t_{p}}\right) I\left(t_{1} \neq t_{2}\right) \ldots I\left(t_{j} \neq t_{j+1}\right) I\left(t_{j+1} \neq t_{j+2}\right) \\
= & h_{b, n, p-1, j}+h_{b, n, p, j+1},
\end{aligned}
$$

where the third equality follows from the fact that $I_{t_{j+1}, b} I_{t_{j+2}, b}=I_{t_{j+1}, b}$ when $t_{j+1}=t_{j+2}$, and we relabel the summation indices. This shows the result of Equation (26) for $b=1,2$, $p \geq 3$ and $j=1, \ldots, p-2$. For $p \geq 2$ and $j=0$, it is easy to see that we also have $h_{b n p 0}=$ $h_{b, n, p-1,0}+h_{b n p 1}$ because

$E\left(\sum_{t=1}^{n} I_{t b}\right)^{p}=E\left(\sum_{t=1}^{n} I_{t b}\right)^{p-1}+\sum_{t_{1}=1}^{n} \sum_{t_{2}=1}^{n} \ldots \sum_{t_{p}=1}^{n} E\left(I_{t_{1}, b} I_{t_{2}, b} \ldots I_{t_{p}, b}\right) I\left(t_{1} \neq t_{2}\right)$.

Therefore, we conclude that the result of Equation (26) holds for $b=1,2, p \geq 2$, and $j=$ $0, \ldots, p-2$.

It follows from Lemma 6 that for $b=1,2, H_{b, p, p-1}=\lim _{n \rightarrow \infty} h_{b, n, p, p-1}$ exists for $p \geq 1$. To show that $H_{b p j}=\lim _{n \rightarrow \infty} h_{b n p j}$ exists for $b=1,2, p \geq 2$ and $j=0, \ldots, p-2$, note that by setting $j=p-2$ in Equation (26), we now have for $b=1,2, p \geq 2$

$h_{b, n, p, p-2}=h_{b, n, p-1, p-2}+h_{b, n, p, p-1}$.

By taking limits, it now follows that $H_{b, p, p-2}$ exists because $H_{b, p, p-2}=H_{b, p-1, p-2}+$ $H_{b, p, p-1}$. Repeating this argument for $j=p-3$, then $p-4$ etc. until $j=0$ then shows that $\lim _{n \rightarrow \infty} h_{b n p j}=H_{b p j}$ exists for $b=1,2, p \geq 2$ and $j=0, \ldots, p-2$. Therefore, the existence 
of $H_{b p j}$ is now shown for $b=1,2, p \geq 1$, and $j=0, \ldots, p-1$. The result of Equation (25) now follows by taking the limit as $n \rightarrow \infty$ in Equation (26).

LEMMA 8. Assume Assumption 1 holds. Then for $p \geq 2$ and $0 \leq j \leq p-1, H_{1 p j}=\Delta^{j} \mu_{p}$ and $H_{2 p j}=\Delta^{j} v_{p}$.

Proof of Lemma 8. Equation (25) states that $H_{b p j}=H_{b, p-1, j}+H_{b, p, j+1}$ for $b=1,2$, $p \geq 2$ and $j=0, \ldots, p-2$. Setting $j+1=i$, we find $H_{b p i}=H_{b, p, i-1}-H_{b, p-1, i-1}=$ $\Delta H_{b, p, i-1}$ for $b=1,2, p \geq 2$ and $i=1, \ldots, p-1$. Repeating this equation gives, if $p \geq 2$ and $1 \leq i-1 \leq p-1, H_{b p i}=\Delta^{2} H_{b, p, i-2}$. Therefore, repeating the equation $k$ times, for $k \geq 0$ and $1 \leq i-k+1 \leq p-1$, we find $H_{b p i}=\Delta^{k} H_{b, p, i-k}$. Setting $k=i$ gives $H_{b p i}=\Delta^{i} H_{b p 0}$ for $p \geq 2$. Therefore, because $\mu_{p}=H_{1 p 0}$ and $v_{p}=H_{2 p 0}, H_{1 p i}=\Delta^{i} \mu_{p}$ and $H_{2 p i}=\Delta^{i} v_{p}$. This completes the proof.

LEMMA 9. Assume Assumption 1 holds. Then for all $p \geq 2$,

$\Delta^{p-1} \mu_{p}=p ! y^{p} 2^{-p / 2} / \Gamma(p / 2+1)$

and

$\Delta^{p-1} v_{p}=p ! y^{p} 2^{p / 2} / \Gamma(p / 2+1)$.

Proof of Lemma 9. The result of Lemma 8 implies that $H_{1, p, p-1}=\Delta^{p-1} \mu_{p}$. Therefore, by Lemma 6, it now follows that

$\Delta^{p-1} \mu_{p}=p ! y^{p} 2^{-p / 2} / \Gamma(p / 2+1)$.

The result for $v_{p}$ is proven analogously. This completes the proof.

LEMMA 10. Assume Assumption 1 holds. Then for the $\mu_{p}$ sequence as defined in Lemma 7 , for $p \geq 2, \mu_{p} \leq(2 p)^{p} \max \left(1, y^{p}\right)$ and $v_{p} \leq 2^{2 p} p^{p} \max \left(1, y^{p}\right)$.

Proof of Lemma 10. By Lemma 7, we have for $p \geq 2$ and $j=0, \ldots, p-2$

$$
H_{1 p j}=H_{1, p-1, j}+H_{1, p, j+1} \leq 2 \max _{i_{1}=0,1} H_{1, p-1+i_{1}, j+i_{1}}
$$

and applying this reasoning $k$ times,

$$
\begin{aligned}
H_{1 p j} & \leq 4 \max _{i_{1}=0,1} \max _{i_{2}=0,1} H_{1, p-2+i_{1}+i_{2}, j+i_{1}+i_{2}} \\
& \leq \cdots \leq 2^{k} \max _{i_{1}=0,1} \ldots \max _{i_{k}=0,1} H_{1, p-k+i_{1}+\cdots+i_{k}, j+i_{1}+\cdots+i_{k} .}
\end{aligned}
$$

Note that Lemma 7 states that $H_{1, p-k+i_{1}+\cdots+i_{k}, j+i_{1}+\cdots+i_{k}}$ is well-defined for $0 \leq j \leq$ $p-k-1$. This is because $H_{b p j}$ is well-defined for $b=1,2, p \geq 1$, and $j=0, \ldots, p-1$ by Lemma 7 , and $p-k+i_{1}+\cdots+i_{k} \geq p-k \geq 1$ and $0 \leq j+i_{1}+\cdots+i_{k} \leq j+k \leq p-1$.

Therefore, setting $j=0$ and $k=p-1$,

$$
\mu_{p}=H_{1 p 0} \leq 2^{p-1} \max _{i_{1}=0,1} \ldots \max _{i_{p-1}=0,1} H_{1,1+i_{1}+\cdots+i_{p-1}, i_{1}+\cdots+i_{p-1}}
$$




$$
=2^{p-1} \max _{0 \leq i \leq p-1} H_{1,1+i, i}=2^{p-1} \max _{1 \leq i \leq p} H_{1, i, i-1}
$$

Because $H_{1, p, p-1}=p ! 2^{-p / 2} y^{p} / \Gamma(p / 2+1)$ by Lemma $6, p ! \leq p^{p}, \Gamma(p / 2+1) \geq 1$ and $2^{-p / 2} \leq 1$ for $p \geq 2$,

$2^{p-1} \max _{1 \leq i \leq p} H_{i, i-1} \leq 2^{p-1} \max \left(1, y^{p}\right) p ! \leq(2 p)^{p} \max \left(1, y^{p}\right)$.

For $v_{p}$ the proof is analogous, except that the upper bound for $v_{p}$ of Lemma 6 is a factor $2^{p}$ larger. This completes the proof.

With the above results in place, we can now complete the proof of Lemma 1:

Proof of Lemma 1. We first apply Lemma 3 to $X_{n}=\sum_{t=1}^{n} I_{t 1}$ for part 1 of Lemma 1, and then to $X_{n}=\sum_{t=1}^{n} I_{t 2}$ for part 2 of Lemma 1. Condition (1) of Lemma 3 holds because $E X_{n}^{p}=E\left(\sum_{t=1}^{n} I_{t 1}\right)^{p}$ converges to $\mu_{p}$ by Lemma 7. Condition (2) of Lemma 3 follows because $\sum_{p=1}^{\infty} \mu_{2 p}^{-1 /(2 p)}=\infty$ because for $p \geq 2, \mu_{2 p} \leq(4 p)^{2 p} \max \left(1, y^{2 p}\right)$ by Lemma 10 . The value for $\mu_{1}$ was calculated in Lemma 6, and the recursive relationship of Equation (8) was shown in Lemma 9. Therefore, the proof of the result for part 1 is now complete. The proof of part 2 of Lemma 1 is analogous.

\section{Proof of Theorem 1}

Proof of Theorem 1. We will show part 1 of Theorem 1, and note that the proof for part 2 is analogous. Under Assumption $1, R_{n}(y)=\sum_{t=1}^{n} I\left(0 \leq x_{t} \leq y n^{-1 / 2}\right)$ converges in distribution to $R(y)$ by Lemma 1 . Therefore, noting that

$P\left(Y_{n 1} \leq y\right)=P\left(n^{1 / 2} \min _{\left\{t: 1 \leq t \leq n, x_{t}>0\right\}} x_{t} \leq y\right)=1-P\left(\sum_{t=1}^{n} I\left(0 \leq x_{t} \leq y n^{-1 / 2}\right) \leq 1 / 2\right)$

and because $1 / 2$ is a continuity point of $R(y)$ for all $y \in \mathbb{R}$,

$L(y)=\lim _{n \rightarrow \infty} P\left(n^{1 / 2} \min _{\left\{t: 1 \leq t \leq n, x_{t}>0\right\}} x_{t} \leq y\right)$

exists for all $y \in \mathbb{R}$. We will verify that $L(y)$ is Lipschitz continuous on $\mathbb{R}$. This follows because, for $y, y^{\prime} \in \mathbb{R}, y \leq y^{\prime}$,

$$
\begin{aligned}
& \left|L(y)-L\left(y^{\prime}\right)\right| \\
& \quad=\lim _{n \rightarrow \infty}\left|P\left(n^{1 / 2} \min _{\left\{t: 1 \leq t \leq n, x_{t}>0\right\}} x_{t} \leq y\right)-P\left(n^{1 / 2} \min _{\left\{t: 1 \leq t \leq n, x_{t}>0\right\}} x_{t} \leq y^{\prime}\right)\right| \\
& =\lim _{n \rightarrow \infty} P\left(y<n^{1 / 2} \min _{\left\{t: 1 \leq t \leq n, x_{t}>0\right\}} x_{t} \leq y^{\prime}\right) \\
& \quad \leq \limsup _{n \rightarrow \infty} P\left(\exists t \in\{1, \ldots, n\}: y<n^{1 / 2} x_{t} \leq y^{\prime}\right) \\
& \quad=\limsup _{n \rightarrow \infty} P\left(\sum_{t=1}^{n} I\left(y n^{-1 / 2} \leq x_{t} \leq y^{\prime} n^{-1 / 2}\right)>1 / 2\right)
\end{aligned}
$$




$$
\begin{aligned}
& \leq 2 \limsup _{n \rightarrow \infty} \sum_{t=1}^{n} P\left(y n^{-1 / 2} \leq x_{t} \leq y^{\prime} n^{-1 / 2}\right) \\
& =2 \limsup _{n \rightarrow \infty} \sum_{t=1}^{n}\left(F_{t}\left(y^{\prime} n^{-1 / 2} t^{-1 / 2}\right)-F_{t}\left(y n^{-1 / 2} t^{-1 / 2}\right)\right) \\
& \leq 2 \limsup _{n \rightarrow \infty} \sum_{t=1}^{n}\left|y-y^{\prime}\right| n^{-1 / 2} t^{-1 / 2} \sup _{t \geq 1} \sup f_{t}(x) \\
& \leq 2 \sup _{t \geq 1} \sup _{x \in \mathbb{R}} f_{t}(x)\left|y-y^{\prime}\right| \sup _{n \geq 1} n^{-1 / 2} \sum_{t=1}^{n} t^{-1 / 2},
\end{aligned}
$$

where the first equality follows from the definition of $L(\cdot)$, the second inequality is the Markov inequality, and the third inequality follows from the mean value theorem.

To show that $Y_{n 1}^{-1}$ converges in distribution, note that for all $z_{1}>0$,

$P\left(Y_{n 1}^{-1} \leq z_{1}\right)=P\left(Y_{n 1} \geq z_{1}^{-1}\right)=1-P\left(Y_{n 1} \leq z_{1}^{-1}\right)$

converges as $n \rightarrow \infty$, and the limit is continuous at any $z_{1}>0$. Furthermore,

$\lim _{z_{1} \rightarrow \infty} \lim _{n \rightarrow \infty} P\left(Y_{n 1}^{-1} \leq z_{1}\right)=1$

because using the reasoning of Equation (27),

$$
\begin{aligned}
& \lim _{z_{1} \rightarrow \infty} \limsup _{n \rightarrow \infty}\left|P\left(Y_{n 1}^{-1} \leq z_{1}\right)-1\right|=\lim _{z_{1} \rightarrow \infty} \limsup _{n \rightarrow \infty}\left|P\left(Y_{n 1} \geq z_{1}^{-1}\right)-P\left(Y_{n 1} \geq 0\right)\right| \\
& \quad \leq \lim _{z_{1} \rightarrow \infty} \limsup _{n \rightarrow \infty} P\left(0 \leq Y_{n 1} \leq z_{1}^{-1}\right) \leq C \lim _{z_{1} \rightarrow \infty} z_{1}^{-1}=0
\end{aligned}
$$

while for $z_{1}<0, P\left(Y_{n 1}^{-1} \leq z_{1}\right)=0$. This implies that $P\left(Y_{n 1}^{-1} \leq z_{1}\right)$ converges to a welldefined limit distribution, and $Y_{n 1}^{-1}$ converges in distribution. The second part of the theorem is proven analogously.

\section{Proof of Lemma 2}

Lemmas 11 to 17 are used for the proof of Lemma 2.

LEMMA 11. A random sequence $X_{n} \in \mathbb{R}^{m}$ converges in distribution to a random variable $X$ if (1) for all $\lambda \neq 0, E\left(\lambda^{\prime} X_{n}\right)^{p}$ converges to a limit $\zeta_{\lambda, p}$ for all $p \in \mathbb{N}$; and (2) $\sum_{p=1}^{\infty} \zeta_{\lambda, 2 p}^{-1 /(2 p)}=\infty$ for all $\lambda \neq 0$

Proof of Lemma 11. Since $\lambda^{\prime} X_{n}$ satisfies the conditions of Lemma 3 for all $\lambda \in \mathbb{R}^{m}$, $\lambda \neq 0$, it follows that $\lambda^{\prime} X_{n} \stackrel{d}{\longrightarrow} Y_{\lambda}$ for some random variable $Y_{\lambda}$ all $\lambda \in \mathbb{R}^{m}, \lambda \neq 0$. Therefore, $E \exp \left(i \lambda^{\prime} X_{n}\right)$ converges pointwise in $\lambda$ to a limit $\psi(\lambda)$, and we only need to show that $Y_{\lambda} \stackrel{d}{=}$ $\lambda^{\prime} X$ for some random variable $X$. It follows from Theorem 2.13 of Van der Vaart (2000, p. 14) that $\psi(\lambda)$ is the characteristic function of a random variable $X$ if $\psi(\lambda)$ is continuous 
at 0 . To show this, note that because $|\exp (i x)-\exp (i y)| \leq|x-y|$ for $x, y \in \mathbb{R}$,

$$
\begin{aligned}
& \left|\psi\left(\lambda_{1}\right)-\psi\left(\lambda_{2}\right)\right| \leq \lim _{n \rightarrow \infty}\left|E \exp \left(i \lambda_{1}^{\prime} X_{n}\right)-E \exp \left(i \lambda_{2}^{\prime} X_{n}\right)\right| \\
& \quad \leq\left|\lambda_{1}-\lambda_{2}\right| \lim _{n \rightarrow \infty} E\left|X_{n}\right|=\left|\lambda_{1}-\lambda_{2}\right| \lim _{n \rightarrow \infty}\left(E X_{n}^{\prime} X_{n}\right)^{1 / 2},
\end{aligned}
$$

and, defining $s_{i}$ as a vector of zeros except for a 1 at spot $i$,

$$
\lim _{n \rightarrow \infty} E X_{n}^{\prime} X_{n}=\lim _{n \rightarrow \infty} \sum_{i=1}^{m} E\left(s_{i}^{\prime} X_{n}\right)^{2}
$$

is well-defined because by assumption, $\lim _{n \rightarrow \infty} E\left(s_{i}^{\prime} X_{n}\right)^{2}=\zeta_{s_{i}, 2}$ is well-defined. Therefore, the lemma is now proven.

LEMMA 12. Assume Assumption 1 holds. Then for $p \geq 2$,

$$
\begin{aligned}
& \lim _{n \rightarrow \infty} \sum_{t_{1}=1}^{n} \ldots \sum_{t_{p}=1}^{n} E\left(I_{t_{1}, y_{1}, 1} I_{t_{2}, y_{2}, 1} \ldots I_{t_{p}, y_{p}, 1}\right) I\left(t_{1}<t_{2}\right) \ldots I\left(t_{p-1}<t_{p}\right) \\
& \quad=2^{-p / 2} \prod_{j=1}^{p} y_{j} / \Gamma(p / 2+1)
\end{aligned}
$$

and

$$
\begin{aligned}
& \lim _{n \rightarrow \infty} \sum_{t_{1}=1}^{n} \ldots \sum_{t_{p}=1}^{n} E\left(I_{t_{1}, y_{1}, 2} I_{t_{2}, y_{2}, 2} \ldots I_{t_{p}, y_{p}, 2}\right) I\left(t_{1}<t_{2}\right) \ldots I\left(t_{p-1}<t_{p}\right) \\
& \quad=2^{p / 2} \prod_{j=1}^{p} y_{j} / \Gamma(p / 2+1) .
\end{aligned}
$$

Proof of Lemma 12. We write that

$$
\begin{aligned}
& E\left(I_{t_{1}, y_{1}, 1} I_{t_{2}, y_{2}, 1} \ldots I_{t_{p}, y_{p}, 1}\right) \\
& =E\left(I\left(0 \leq x_{t_{1}} \leq n^{-1 / 2} y_{1}\right) \ldots I\left(0 \leq x_{t_{p}} \leq n^{-1 / 2} y_{p}\right)\right) \\
& =\int \ldots \int I\left(0 \leq z_{1}+z_{2}+\cdots+z_{p} \leq n^{-1 / 2} y_{p}\right) \ldots I\left(0 \leq z_{1} \leq n^{-1 / 2} y_{1}\right) \\
& \quad \times g_{t_{1}}\left(z_{1}\right) g_{t_{2}-t_{1}}\left(z_{2}\right) \ldots g_{t_{p}-t_{p-1}}\left(z_{p}\right) d z_{p} \ldots d z_{1} .
\end{aligned}
$$

We follow a similar argument as in the proof of Lemma 6 and approximate $g_{t_{1}}(z)$ by $t_{1}^{-1 / 2} \phi(0)$ and $g_{t_{j}-t_{j-1}}(z)$ by $\left(t_{j}-t_{j-1}\right)^{-1 / 2} \phi(0)$ for $j=2,3, \ldots, p$ and write that

$$
\begin{aligned}
& \lim _{n \rightarrow \infty} \sum_{t_{1}=1}^{n} \ldots \sum_{t_{p}=1}^{n} I\left(t_{1}<t_{2}\right) I\left(t_{2}<t_{3}\right) \ldots I\left(t_{p-1}<t_{p}\right) E\left(I_{t_{1}, y_{1}, 1} \ldots I_{t_{p}, y_{p}, 1}\right) \\
& \quad=\lim _{n \rightarrow \infty} \sum_{t_{1}=1}^{n} \ldots \sum_{t_{p}=1}^{n} I\left(t_{1}<t_{2}\right) \ldots I\left(t_{p-1}<t_{p}\right)
\end{aligned}
$$




$$
\begin{aligned}
& \times \int \ldots \int I\left(0 \leq z_{1}+\cdots+z_{p} \leq n^{-1 / 2} y_{p}\right) \ldots I\left(0 \leq z_{1} \leq n^{-1 / 2} y_{1}\right) \\
& \times \phi(0)^{p} t_{1}^{-1 / 2}\left(t_{2}-t_{1}\right)^{-1 / 2} \ldots\left(t_{p}-t_{p-1}\right)^{-1 / 2} d z_{p} \ldots d z_{1} .
\end{aligned}
$$

Since

$$
\int \cdots \int I\left(0 \leq z_{1}+\cdots+z_{p} \leq n^{-1 / 2} y_{p}\right) \ldots I\left(0 \leq z_{1} \leq n^{-1 / 2} y_{1}\right) d z_{p} \ldots d z_{1}=n^{-p / 2} \prod_{j=1}^{p} y_{j},
$$

we have

$$
\begin{aligned}
\lim _{n \rightarrow \infty} & \sum_{t_{1}=1}^{n} \ldots \sum_{t_{p}=1}^{n} I\left(t_{1}<t_{2}\right) I\left(t_{2}<t_{3}\right) \ldots I\left(t_{p-1}<t_{p}\right) E\left(I_{t_{1}, y_{1}, 1} \ldots I_{t_{p}, y_{p}, 1}\right) \\
= & \phi(0)^{p} \prod_{j=1}^{p} y_{j} \lim _{n \rightarrow \infty} n^{-p / 2} \sum_{t_{1}=1}^{n} \ldots \sum_{t_{p}=1}^{n} I\left(t_{1}<t_{2}\right) \ldots \\
& \times I\left(t_{p-1}<t_{p}\right) t_{1}^{-1 / 2}\left(t_{2}-t_{1}\right)^{-1 / 2} \ldots\left(t_{p}-t_{p-1}\right)^{-1 / 2}
\end{aligned}
$$

Lemmas 4 and 5 now imply, by noting that $\phi(0)^{p}=(2 \pi)^{-p / 2}$ and $(\Gamma(1 / 2))^{p}=\pi^{p / 2}$, that

$$
\begin{aligned}
& \lim _{n \rightarrow \infty} \sum_{t_{1}=1}^{n} \ldots \sum_{t_{p}=1}^{n} E\left(I_{t_{1}, y_{1}, 1} I_{t_{2}, y_{2}, 1} \ldots I_{t_{p}, y_{p}, 1}\right) I\left(t_{1}<t_{2}\right) \ldots I\left(t_{p-1}<t_{p}\right) \\
& \quad=\phi(0)^{p} \prod_{j=1}^{p} y_{j} \int_{0}^{1} \int_{0}^{s_{p}} \ldots \int_{0}^{s_{2}} s_{1}^{-1 / 2}\left(s_{2}-s_{1}\right)^{-1 / 2} \ldots\left(s_{p}-s_{p-1}\right)^{-1 / 2} d s_{1} d s_{2} \ldots d s_{p} \\
& \quad=2^{-p / 2} \prod_{j=1}^{p} y_{j} / \Gamma(p / 2+1) .
\end{aligned}
$$

The proof of the second result of the lemma is analogous, except that the equivalent of Equation (28) now receives an additional $2^{p}$ factor.

LEMMA 13. Assume Assumption 1 holds. Then we have

$\lim _{n \rightarrow \infty} E\left(\sum_{t_{1}=1}^{n} I_{t_{1}, y_{1}, 1}\right)=y_{1} \sqrt{2 / \pi}$

and

$$
\lim _{n \rightarrow \infty} E\left(\sum_{t_{1}=1}^{n} I_{t_{1}, y_{1}, 2}\right)=2 y_{1} \sqrt{2 / \pi}
$$

and for $p \geq 2$,

$$
\lim _{n \rightarrow \infty} \sum_{t_{1}=1}^{n} \sum_{t_{2}=1}^{n} \ldots \sum_{t_{p}=1}^{n} E\left(I_{t_{1}, y_{1}, 1} I_{t_{2}, y_{2}, 1} \ldots I_{t_{p}, y_{p}, 1}\right) I\left(t_{1} \neq t_{2}\right) I\left(t_{2} \neq t_{3}\right) \ldots I\left(t_{p-1} \neq t_{p}\right)
$$




$$
=p ! 2^{-p / 2} \prod_{j=1}^{p} y_{j} / \Gamma(p / 2+1)
$$

and

$$
\begin{aligned}
& \lim _{n \rightarrow \infty} \sum_{t_{1}=1}^{n} \sum_{t_{2}=1}^{n} \ldots \sum_{t_{p}=1}^{n} E\left(I_{t_{1}, y_{1}, 2} I_{t_{2}, y_{2}, 2} \ldots I_{t_{p}, y_{p}, 2}\right) I\left(t_{1} \neq t_{2}\right) I\left(t_{2} \neq t_{3}\right) \ldots I\left(t_{p-1} \neq t_{p}\right) \\
& \quad=p ! 2^{p / 2} \prod_{j=1}^{p} y_{j} / \Gamma(p / 2+1) .
\end{aligned}
$$

Proof of Lemma 13. The first two assertions of the lemma follows from Lemma 6 when we set $y=y_{1}$. The third result follows immediately from noting that

$$
\begin{aligned}
& \lim _{n \rightarrow \infty} \sum_{t_{1}=1}^{n} \sum_{t_{2}=1}^{n} \ldots \sum_{t_{p}=1}^{n} E\left(I_{t_{1}, y_{1}, 1} I_{t_{2}, y_{2}, 1} \ldots I_{t_{p}, y_{p}, 1}\right) I\left(t_{1} \neq t_{2}\right) I\left(t_{2} \neq t_{3}\right) \ldots I\left(t_{p-1} \neq t_{p}\right) \\
& \quad=p ! 2^{-p / 2} \prod_{j=1}^{p} y_{j} / \Gamma(p / 2+1),
\end{aligned}
$$

because there are $p$ ! orderings of $\left\{t_{1}, t_{2}, \ldots, t_{p}\right\}$, and Lemma 12 ensures that any possible ordering of $\left\{y_{1}, y_{2}, \ldots, y_{p}\right\}$ gives the same limit result. The fourth result follows analogously.

LEMMA 14. Assume Assumption 1 holds. For $b=1,2, p \geq 2$ and $j=1,2, \ldots, p-1$, define

$$
\begin{aligned}
& h_{b n p j}\left(y_{1}, y_{2}, \ldots, y_{p}\right) \\
& \quad=\sum_{t_{1}=1}^{n} \sum_{t_{2}=1}^{n} \ldots \sum_{t_{p}=1}^{n} E\left(I_{t_{1}, y_{1}, b} I_{t_{2}, y_{2}, b} \ldots I_{t_{p}, y_{p}, b}\right) I\left(t_{1} \neq t_{2}\right) I\left(t_{2} \neq t_{3}\right) \ldots I\left(t_{j} \neq t_{j+1}\right),
\end{aligned}
$$

and for $b=1,2$ and $p \geq 1$, define

$h_{b n p 0}\left(y_{1}, y_{2}, \ldots, y_{p}\right)=\sum_{t_{1}=1}^{n} \sum_{t_{2}=1}^{n} \ldots \sum_{t_{p}=1}^{n} E\left(I_{t_{1}, y_{1}, b} I_{t_{2}, y_{2}, b} \ldots I_{t_{p}, y_{p}, b}\right)$.

Then $H_{b p j}\left(y_{1}, y_{2}, \ldots, y_{p}\right)=\lim _{n \rightarrow \infty} h_{b n p j}\left(y_{1}, y_{2}, \ldots, y_{p}\right)$ is well-defined for $b=1,2, p \geq 1$ and $j=0,1, \ldots, p-1$, and as a consequence,

$\mu_{p}\left(y_{1}, y_{2}, \ldots, y_{p}\right)=H_{1 p 0}\left(y_{1}, \ldots, y_{p}\right)=\lim _{n \rightarrow \infty} h_{1 n p 0}\left(y_{1}, y_{2}, \ldots, y_{p}\right)$

and

$v_{p}\left(y_{1}, y_{2}, \ldots, y_{p}\right)=H_{2 p 0}\left(y_{1}, \ldots, y_{p}\right)=\lim _{n \rightarrow \infty} h_{2 n p 0}\left(y_{1}, y_{2}, \ldots, y_{p}\right)$

are well-defined for $p \geq 1$. Also, for $b=1,2, p \geq 2, j=0,1, \ldots, p-2$ and $0 \leq y_{1} \leq y_{2} \leq$ $\cdots \leq y_{p}$

$H_{b p j}\left(y_{1}, y_{2}, \ldots, y_{p}\right)=H_{b, p-1, j}\left(y_{1}, \ldots, y_{j+1}, y_{j+3}, \ldots, y_{p}\right)+H_{b, p, j+1}\left(y_{1}, y_{2}, \ldots, y_{p}\right)$. 
Proof of Lemma 14. For $b=1,2, p \geq 3$ and $j=1,2, \ldots, p-2$, we can write

$$
\begin{aligned}
h_{b n p j}\left(y_{1}, y_{2}, \ldots, y_{p}\right) \\
=\sum_{t_{1}=1}^{n} \sum_{t_{2}=1}^{n} \ldots \sum_{t_{p}=1}^{n} E\left(I_{t_{1}, y_{1}, b} I_{t_{2}, y_{2}, b} \ldots I_{t_{p}, y_{p}, b}\right) I\left(t_{1} \neq t_{2}\right) \ldots I\left(t_{j} \neq t_{j+1}\right) \\
=\sum_{t_{1}=1}^{n} \sum_{t_{2}=1}^{n} \ldots \sum_{t_{p}=1}^{n} E\left(I_{t_{1}, y_{1}, b} I_{t_{2}, y_{2}, b} \ldots I_{t_{p}, y_{p}, b}\right) I\left(t_{1} \neq t_{2}\right) \ldots I\left(t_{j} \neq t_{j+1}\right) \\
\quad \times\left(I\left(t_{j+1}=t_{j+2}\right)+I\left(t_{j+1} \neq t_{j+2}\right)\right) \\
=\sum_{t_{1}=1}^{n} \sum_{t_{2}=1}^{n} \ldots \sum_{t_{p-1}=1}^{n} E\left(I_{t_{1}, y_{1}, b} \ldots I_{t_{j}, y_{j}, b} I_{t_{j+1}, y_{j+1}, b} I_{t_{j+2}, y_{j+3}, b} \ldots I_{t_{p-1}, y_{p}, b}\right) \\
\quad \times I\left(t_{1} \neq t_{2}\right) \ldots I\left(t_{j} \neq t_{j+1}\right) \\
\quad+\sum_{t_{1}=1}^{n} \sum_{t_{2}=1}^{n} \ldots \sum_{t_{p}=1}^{n} E\left(I_{t_{1}, y_{1}, b} I_{t_{2}, y_{2}, b} \ldots I_{t_{p}, y_{p}, b}\right) I\left(t_{1} \neq t_{2}\right) \ldots I\left(t_{j} \neq t_{j+1}\right) I\left(t_{j+1} \neq t_{j+2}\right) \\
=h_{b, n, p-1, j}\left(y_{1}, y_{2}, \ldots, y_{j+1}, y_{j+3}, \ldots, y_{p}\right)+h_{b, n, p, j+1}\left(y_{1}, y_{2}, \ldots, y_{p}\right),
\end{aligned}
$$

where the third equality follows from the fact that $I_{t_{j+1}, y_{j+1}, b} I_{t_{j+2}, y_{j+2}, b}=I_{t_{j+1}, y_{j+1}, b}$ $I_{t_{j+1}, y_{j+2}, b}=I_{t_{j+1}}, \min \left(y_{j+1}, y_{j+2}\right), b=I_{t_{j+1}, y_{j+1}, b}$ when $t_{j+1}=t_{j+2}$ and from relabeling the summation indices. This shows the result of Equation (30) for $b=1,2, p \geq 3$, and $j=$ $1,2, \ldots, p-2$. Similar to the argument in the proof of Lemma 7, for $b=1,2, p \geq 2$, and $j=0$, we also have $h_{b n p 0}\left(y_{1}, \ldots, y_{p}\right)=h_{b, n, p-1,0}\left(y_{1}, y_{3} \ldots, y_{p}\right)+h_{b n p 1}\left(y_{1}, \ldots, y_{p}\right)$. Therefore, we conclude that the result of Equation (30) holds for $b=1,2, p \geq 2$, and $j=0, \ldots, p-2$. It follows from Lemma 13 that for $b=1,2$,

$H_{b, p, p-1}\left(y_{1}, y_{2}, \ldots, y_{p}\right)=\lim _{n \rightarrow \infty} h_{b, n, p, p-1}\left(y_{1}, y_{2}, \ldots, y_{p}\right)$

exists for $p \geq 1$. To show that $H_{b p j}\left(y_{1}, y_{2}, \ldots, y_{p}\right)=\lim _{n \rightarrow \infty} h_{b n p j}\left(y_{1}, y_{2}, \ldots, y_{p}\right)$ exists for $b=1,2, p \geq 2$ and $j=0, \ldots, p-2$, note that by setting $j=p-2$ in Equation (30), we now have for $b=1,2$ and $p \geq 2$

$h_{b, n, p, p-2}\left(y_{1}, y_{2}, \ldots, y_{p}\right)=h_{b, n, p-1, p-2}\left(y_{1}, y_{2}, \ldots, y_{p-1}\right)+h_{b, n, p, p-1}\left(y_{1}, y_{2}, \ldots, y_{p}\right)$.

By taking limits, it now follows that $H_{b, p, p-2}\left(y_{1}, y_{2}, \ldots, y_{p-1}\right)$ exists because

$H_{b, p, p-2}\left(y_{1}, y_{2}, \ldots, y_{p}\right)=H_{b, p-1, p-2}\left(y_{1}, y_{2}, \ldots, y_{p-1}\right)+H_{b, p, p-1}\left(y_{1}, y_{2}, \ldots, y_{p}\right)$.

Repeating this argument for $j=p-3$, then $p-4$ etc. until $j=0$ then shows that $\lim _{n \rightarrow \infty} h_{b n p j}\left(y_{1}, y_{2}, \ldots, y_{p}\right)=H_{b p j}\left(y_{1}, \ldots, y_{p}\right)$ exists for $b=1,2, p \geq 2$, and $j=0, \ldots, p-$ 2. Therefore, the existence of $H_{b p j}\left(y_{1}, \ldots, y_{p}\right)$ is now shown for $b=1,2, p \geq 1$, and $j=0, \ldots, p-1$. The result of Equation (29) now follows by taking the limit as $n \rightarrow \infty$ in Equation (30).

LEMMA 15. Assume Assumption 1 holds. Then for the $\mu_{p}\left(y_{1}, y_{2}, \ldots, y_{p}\right)$ sequence defined in Lemma 14, we have for $p \geq 2$ and $y_{1} \leq y_{2} \leq \cdots \leq y_{p}, \mu_{p}\left(y_{1}, y_{2}, \ldots, y_{p}\right) \leq$ $(2 p)^{p} \max \left(1, y_{p}^{p}\right)$. For the $v_{p}\left(y_{1}, y_{2}, \ldots, y_{p}\right)$ sequence defined in Lemma 14, we have for $p \geq 2$ and $y_{1} \leq y_{2} \leq \cdots \leq y_{p}, v_{p}\left(y_{1}, y_{2}, \ldots, y_{p}\right) \leq 2^{2 p} p^{p} \max \left(1, y_{p}^{p}\right)$. 
Proof of Lemma 15. Note that $H_{b p j}\left(y_{1}, y_{2}, \ldots, y_{p}\right)$ is increasing in each argument $y_{j}$ for $j=1,2, \ldots, p$. Therefore, since $y_{1} \leq y_{2} \leq \cdots \leq y_{p}$

$H_{1 p j}\left(y_{1}, y_{2}, \ldots, y_{p}\right) \leq H_{1 p j}\left(y_{p}, \ldots, y_{p}\right) \leq(2 p)^{p} \max \left(1, y_{p}^{p}\right)$,

where the last inequality follows from Lemma 10 and setting $y=y_{p}$. The argument for $v_{p}\left(y_{1}, y_{2}, \ldots, y_{p}\right)$ is analogous.

LEMMA 16. Assume Assumption 1 holds. Define

$X_{n 1}=\left(\sum_{t=1}^{n} I_{t, y_{1}, 1}, \sum_{t=1}^{n} I_{t, y_{2}, 1}, \ldots, \sum_{t=1}^{n} I_{t, y_{m}, 1}\right)^{\prime}$

and

$X_{n 2}=\left(\sum_{t=1}^{n} I_{t, y_{1}, 2}, \sum_{t=1}^{n} I_{t, y_{2}, 2}, \ldots, \sum_{t=1}^{n} I_{t, y_{m}, 2}\right)^{\prime}$

Then, for all $p>1, \mu_{\lambda p}=\lim _{n \rightarrow \infty} E\left(\lambda^{\prime} X_{n 1}\right)^{p}$ and $\nu_{\lambda p}=\lim _{n \rightarrow \infty} E\left(\lambda^{\prime} X_{n 2}\right)^{p}$ are welldefined.

Proof of Lemma 16. For the first case, by using the definition of $\mu_{p}\left(y_{1}, y_{2}, \ldots, y_{p}\right)$ in Lemma 14, we write that

$$
\begin{aligned}
& \lim _{n \rightarrow \infty} E\left(\lambda^{\prime} X_{n 1}\right)^{p} \\
& \quad=\sum_{j_{1}=1}^{m} \sum_{j_{2}=1}^{m} \ldots \sum_{j_{p}=1}^{m} \lambda_{j_{1}} \lambda_{j_{2}} \ldots \lambda_{j_{p}} \lim _{n \rightarrow \infty} \sum_{t_{1}=1}^{n} \sum_{t_{2}=1}^{n} \ldots \sum_{t_{p}=1}^{n} E\left(I_{t_{1}, y_{j_{1}, 1}} I_{t_{2}, y_{j_{2}}, 1} \ldots I_{t_{p}, y_{j_{p}, 1}}\right) \\
& =\sum_{j_{1}=1}^{m} \sum_{j_{2}=1}^{m} \ldots \sum_{j_{p}=1}^{m} \lambda_{j_{1}} \lambda_{j_{2}} \ldots \lambda_{j_{p}} \mu_{p}\left(y_{j_{1}}, y_{j_{2}}, \ldots, y_{j_{p}}\right) .
\end{aligned}
$$

Since $\mu_{p}\left(y_{j_{1}}, y_{j_{2}}, \ldots, y_{j_{p}}\right)$ is well-defined by Lemma $14, \lim _{n \rightarrow \infty} E\left(\lambda^{\prime} X_{n 1}\right)^{p}$ is also welldefined. The second case is analogous, but uses the definition of $v_{p}\left(y_{1}, y_{2}, \ldots, y_{p}\right)$ instead of $\mu_{p}\left(y_{1}, y_{2}, \ldots, y_{p}\right)$ from Lemma 14 .

LEMMA 17. Assume Assumption 1 holds, and let $\mu_{\lambda p}$ and $v_{\lambda p}$ be as defined in Lemma 16. Then for $p \geq 2$,

$$
\left|\mu_{\lambda p}\right| \leq(2 m p)^{p} \max _{1 \leq i \leq m}\left|\lambda_{i}\right|^{p} \max \left(1, y_{p}^{p}\right)
$$

and

$$
\left|v_{\lambda p}\right| \leq 2^{2 p}(m p)^{p} \max _{1 \leq i \leq m}\left|\lambda_{i}\right|^{p} \max \left(1, y_{p}^{p}\right)
$$


Proof of Lemma 17. Since $\mu_{p}\left(y_{1}, \ldots, y_{p}\right) \leq(2 p)^{p} \max \left(1, y_{p}^{p}\right)$ by Lemma 15 , it follows from Equation (31) that

$$
\begin{aligned}
\left|\mu_{\lambda p}\right| & \leq \sum_{j_{1}=1}^{m} \sum_{j_{2}=1}^{m} \ldots \sum_{j_{p}=1}^{m}\left|\lambda_{j_{1}}\right|\left|\lambda_{j_{2}}\right| \ldots\left|\lambda_{j_{p}}\right| \mu_{p}\left(y_{j_{1}}, y_{j_{2}}, \ldots, y_{j_{p}}\right) \\
& \leq \sum_{j_{1}=1}^{m} \sum_{j_{2}=1}^{m} \ldots \sum_{j_{p}=1}^{m} \max _{1 \leq i \leq m}\left|\lambda_{i}\right|^{p}(2 p)^{p} \max \left(1, y_{p}^{p}\right) \\
& =(2 m p)^{p} \max _{1 \leq i \leq m}\left|\lambda_{i}\right|^{p} \max \left(1, y_{p}^{p}\right) .
\end{aligned}
$$

The second part is shown analogously, except that the upper bound for $v_{p}\left(y_{1}, \ldots, y_{p}\right)$ from Lemma 15 is now used.

We now provide the proof of Lemma 2.

Proof of Lemma 2. We first apply Lemma 11 to

$X_{n 1}=\left(\sum_{t=1}^{n} I_{t, y_{1}, 1}, \sum_{t=1}^{n} I_{t, y_{2}, 1}, \ldots, \sum_{t=1}^{n} I_{t, y_{m}, 1}\right)^{\prime}$

for part 1 of Lemma 2, and then to

$X_{n 2}=\left(\sum_{t=1}^{n} I_{t, y_{1}, 2}, \sum_{t=1}^{n} I_{t, y_{2}, 2}, \ldots, \sum_{t=1}^{n} I_{t, y_{m}, 2}\right)^{\prime}$

for part 2 of Lemma 2. Condition (1) of Lemma 11 holds because $E\left(\lambda^{\prime} X_{n 1}\right)^{p}$ converges to $\mu_{\lambda p}$ by Lemma 16. Condition (2) of Lemma 11 follows because $\sum_{p=1}^{\infty} \mu_{\lambda, 2 p}^{-1 /(2 p)}=$ $\infty$ because for $p \geq 2, \mu_{\lambda, 2 p} \leq(4 m p)^{2 p} \max _{1 \leq i \leq m}\left(1, \lambda_{i}^{2 p}\right) \max \left(1, y_{p}^{2 p}\right)$ by Lemma 17 . Therefore, the proof of the result for part 1 is now complete. The proof of part 2 of Lemma 2 is analogous.

\section{Proof of Theorem 2}

Theorem 2 relies on Lemma 18.

LEMMA 18. For all $\left(y_{1}, \ldots, y_{m}\right)^{\prime} \in \mathbb{R}^{m}$ and $\left(y_{1}^{\prime}, \ldots, y_{m}^{\prime}\right)^{\prime} \in \mathbb{R}^{m}$,

$$
\begin{aligned}
& \mid P\left(Y_{n i} \leq y_{i} \text { for } i=1, \ldots, m\right)-P\left(Y_{n i} \leq y_{i}^{\prime} \text { for } i=1, \ldots, m\right) \mid \\
& \quad \leq \sum_{i=1}^{m}\left|P\left(Y_{n i} \leq y_{i}\right)-P\left(Y_{n i} \leq y_{i}^{\prime}\right)\right| .
\end{aligned}
$$

Proof of Lemma 18. The result follows because

$\mid P\left(Y_{n i} \leq y_{i}\right.$ for $\left.i=1, \ldots, m\right)-P\left(Y_{n i} \leq y_{i}^{\prime}\right.$ for $\left.i=1, \ldots, m\right) \mid$ 


$$
\begin{aligned}
&\left|E \prod_{i=1}^{m}\left(I\left(Y_{n i} \leq y_{i}\right)-I\left(Y_{n i} \leq y_{i}^{\prime}\right)\right)\right| \\
& \leq E \prod_{i=1}^{m}\left|I\left(Y_{n i} \leq y_{i}\right)-I\left(Y_{n i} \leq y_{i}^{\prime}\right)\right| \\
& \leq E \sum_{i=1}^{m}\left|I\left(Y_{n i} \leq y_{i}\right)-I\left(Y_{n i} \leq y_{i}^{\prime}\right)\right| \\
&=\sum_{i=1}^{m} E I\left(\min \left(y_{i}, y_{i}^{\prime}\right) \leq Y_{n i} \leq \max \left(y_{i}, y_{i}^{\prime}\right)\right) \\
&=\sum_{i=1}^{m}\left(P\left(Y_{n i} \leq \max \left(y_{i}, y_{i}^{\prime}\right)\right)-P\left(Y_{n i} \leq \min \left(y_{i}, y_{i}^{\prime}\right)\right)\right) \\
&=\sum_{i=1}^{m}\left|P\left(Y_{n i} \leq y_{i}\right)-P\left(Y_{n i} \leq y_{i}^{\prime}\right)\right| .
\end{aligned}
$$

We can now complete the proof of Theorem 2.

Proof of Theorem 2. We first show the first part of Theorem 2. Under Assumption 1, $\left(\sum_{t=1}^{n} I\left(0 \leq x_{t} \leq y_{1} n^{-1 / 2}\right), \sum_{t=1}^{n} I\left(0 \leq x_{t} \leq y_{2} n^{-1 / 2}\right), \ldots, \sum_{t=1}^{n} I\left(0 \leq x_{t} \leq y_{m} n^{-1 / 2}\right)\right)^{\prime}$ converges in distribution to $\left(R\left(y_{1}\right), R\left(y_{2}\right), \ldots, R\left(y_{m}\right)\right)^{\prime}$ by Lemma 2 . We have

$P\left(Y_{n i} \leq y_{i}\right.$ for $\left.i=1, \ldots, m\right)=P\left(\sum_{t=1}^{n} I\left(0<x_{t} \leq y_{i} n^{-1 / 2}\right) \geq i-1 / 2\right.$ for $\left.i=1, \ldots, m\right)$

and the last expression converges to some function $L\left(y_{1}, \ldots, y_{m}\right)$ since $(1 / 2,3 / 2, \ldots, m-$ $1 / 2)$ is a continuity point of the distribution of $\left(R\left(y_{1}\right), \ldots, R\left(y_{m}\right)\right)^{\prime}$. By Lemma 18, we have

$$
\begin{aligned}
& \left|L\left(y_{1}, \ldots, y_{m}\right)-L\left(y_{1}^{\prime}, \ldots, y_{m}^{\prime}\right)\right| \\
& \quad=\lim _{n \rightarrow \infty} \mid P\left(Y_{n i} \leq y_{i} \text { for } i=1, \ldots, m\right)-P\left(Y_{n i} \leq y_{i}^{\prime} \text { for } i=1, \ldots, m\right) \mid \\
& \quad \leq \sum_{i=1}^{m} \lim _{n \rightarrow \infty}\left|P\left(Y_{n i} \leq y_{i}\right)-P\left(Y_{n i} \leq y_{i}^{\prime}\right)\right| \\
& \quad \leq \sum_{i=1}^{m} \lim _{n \rightarrow \infty} P\left(\exists t \in\{1, \ldots, n\}: \min \left(y_{i}, y_{i}^{\prime}\right)<n^{1 / 2} x_{t} \leq \max \left(y_{i}, y_{i}^{\prime}\right)\right) \\
& \quad \leq C \sum_{i=1}^{m}\left|y_{i}-y_{i}^{\prime}\right|,
\end{aligned}
$$

where the last inequality follows from the reasoning of Equation (27). To show that $\left(Y_{n 1}^{-1}, \ldots, Y_{n m}^{-1}\right)^{\prime}$ converges in distribution, note that if $z_{i}>0$ for all $i$,

$P\left(Y_{n i}^{-1} \leq z_{i}\right.$ for $\left.i=1, \ldots, m\right)=P\left(Y_{n i} \geq z_{i}^{-1}\right.$ for $\left.i=1, \ldots, m\right)$ 
converges as $n \rightarrow \infty$ and is continuous at $\left(z_{1}, \ldots, z_{L}\right)^{\prime}$. Also, using the reasoning of Equation (32),

$$
\begin{aligned}
& \limsup _{n \rightarrow \infty} \mid P\left(Y_{n i}^{-1} \leq z_{i} \text { for } i=1, \ldots, m\right)-1 \mid \\
& \quad=\limsup _{n \rightarrow \infty} \mid P\left(Y_{n i} \geq z_{i}^{-1} \text { for } i=1, \ldots, m\right)-P\left(Y_{n i} \geq 0 \text { for } i=1, \ldots, m\right) \mid \\
& \quad \leq \limsup _{n \rightarrow \infty} \sum_{i=1}^{m} P\left(0 \leq Y_{n i} \leq z_{i}^{-1}\right) \leq C \sum_{i=1}^{m} z_{i}^{-1}
\end{aligned}
$$

which converges to 0 if $z_{i} \rightarrow \infty$ for all $i$. Therefore, $\lim _{n \rightarrow \infty} P\left(Y_{n i}^{-1} \leq z_{i}\right.$ for $\left.i=1, \ldots, m\right)$ converges to 1 if $z_{i} \rightarrow \infty$ for all $i$. Together with the observation that $P\left(Y_{n i}^{-1} \leq z_{i}\right.$ for $i=$ $1, \ldots, m)=0$ if $z_{i}<0$, this implies that $P\left(Y_{n 1}^{-1} \leq z_{1}, \ldots, Y_{n m}^{-1} \leq z_{m}\right)$ converges to a welldefined limit distribution. Therefore, $\left(Y_{n 1}^{-1}, \ldots, Y_{n m}^{-1}\right)^{\prime}$ converges in distribution. The second part of the theorem is proven analogously.

\section{Proof of Theorem 3}

Lemma 19 is key to the proof of Theorem 3. The proof of Lemma 19 is taken from Pötscher (2013, Thm. 1) and is stated here for completeness.

LEMMA 19. Assume Assumption 1 holds. Then for $q>1$,

$n^{-q / 2} \sum_{t=1}^{n} x_{t}^{-q} I\left(x_{t}>0\right)=O_{p}(1)$

and

$n^{-q / 2} \sum_{t=1}^{n}\left|x_{t}\right|^{-q}=O_{p}(1)$.

Proof of Lemma 19. First note that, for all $\delta>0$,

$$
\begin{aligned}
& n^{-q / 2} \sum_{t=1}^{n} x_{t}^{-q} I\left(x_{t}>0\right) \\
& =n^{-q / 2} \sum_{t=1}^{n} x_{t}^{-q} I\left(0<x_{t} \leq \delta n^{-1 / 2}\right)+n^{-q / 2} \sum_{t=1}^{n} x_{t}^{-q} I\left(x_{t}>\delta n^{-1 / 2}\right) .
\end{aligned}
$$

Now

$$
\begin{aligned}
& E n^{-q / 2} \sum_{t=1}^{n} x_{t}^{-q} I\left(x_{t}>\delta n^{-1 / 2}\right)=n^{-q / 2} \sum_{t=1}^{n} t^{-q / 2} E\left(t^{-1 / 2} x_{t}\right)^{-q} I\left(t^{-1 / 2} x_{t}>\delta n^{-1 / 2} t^{-1 / 2}\right) \\
& =n^{-q / 2} \sum_{t=1}^{n} t^{-q / 2} \int_{t^{-1 / 2} n^{-1 / 2} \delta}^{\infty} x^{-q} f_{t}(x) d x
\end{aligned}
$$




$$
\begin{aligned}
& \leq \sup _{t \geq 1, x \in \mathbb{R}} f_{t}(x) n^{-q / 2} \sum_{t=1}^{n} t^{-q / 2} \int_{t^{-1 / 2} n^{-1 / 2} \delta}^{\infty} x^{-q} d x \\
& =\sup _{t \geq 1, x \in \mathbb{R}} f_{t}(x) n^{-q / 2} \sum_{t=1}^{n} t^{-q / 2}(1 /(1-q))\left[x^{-q+1}\right]_{t^{-1 / 2} n^{-1 / 2} \delta}^{\infty} \\
& =\sup _{t \geq 1, x \in \mathbb{R}} f_{t}(x) n^{-q / 2} \sum_{t=1}^{n} t^{-q / 2}(1 /(q-1))\left(t^{-1 / 2} n^{-1 / 2} \delta\right)^{-q+1} \\
& =\sup _{t \geq 1, x \in \mathbb{R}} f_{t}(x) \sum_{t=1}^{n}(1 /(q-1)) t^{-1 / 2} n^{-1 / 2} \delta^{-q+1} .
\end{aligned}
$$

In addition, for all $\delta>0$,

$$
\begin{aligned}
& P\left(n^{-q / 2} \sum_{t=1}^{n} x_{t}^{-q} I\left(0<x_{t}<\delta n^{-1 / 2}\right)>0\right) \\
& \quad=P\left(\exists t \in\{1, \ldots, n\}: 0<x_{t}<\delta n^{-1 / 2}\right) \leq \sum_{t=1}^{n} P\left(0<x_{t}<\delta n^{-1 / 2}\right) \\
& \quad=\sum_{t=1}^{n} \int_{0}^{\delta n^{-1 / 2} t^{-1 / 2}} f_{t}(x) d x \\
& \quad \leq \sup _{t \geq 1, x \in \mathbb{R}} f_{t}(x) \sum_{t=1}^{n} \delta n^{-1 / 2} t^{-1 / 2} .
\end{aligned}
$$

Therefore,

$$
\begin{aligned}
& \limsup _{n \rightarrow \infty} P\left(n^{-q / 2} \sum_{t=1}^{n} x_{t}^{-q} I\left(x_{t}>0\right)>K\right) \\
& \leq \limsup _{n \rightarrow \infty} P\left(n^{-q / 2} \sum_{t=1}^{n} x_{t}^{-q} I\left(0<x_{t}<\delta n^{-1 / 2}\right)>0\right) \\
& \quad+\limsup _{n \rightarrow \infty} P\left(n^{-q / 2} \sum_{t=1}^{n} x_{t}^{-q} I\left(x_{t}>\delta n^{-1 / 2}\right)>K\right) \\
& \leq \sup _{t \geq 1, x \in \mathbb{R}_{t}} f_{t}(x) \limsup _{n \rightarrow \infty} \sum_{t=1}^{n} \delta n^{-1 / 2} t^{-1 / 2} \\
& \quad+K^{-1} \sup _{t \geq 1, x \in \mathbb{R}^{\prime}} f_{t}(x) \limsup _{n \rightarrow \infty} \sum_{t=1}^{n}(1 /(q-1)) t^{-1 / 2} n^{-1 / 2} \delta^{-q+1} \\
& \leq C_{1} \delta+C_{2} K^{-1} \delta^{-q+1}
\end{aligned}
$$


for constants $C_{1}$ and $C_{2}$ independent of $n$, and the second inequality follows from the Markov inequality. Therefore,

$\underset{K \rightarrow \infty}{\limsup \limsup _{n \rightarrow \infty} P}\left(n^{-q / 2} \sum_{t=1}^{n} x_{t}^{-q} I\left(x_{t}>0\right)>K\right) \leq C_{1} \delta$,

and since $\delta$ was arbitrary, the result now follows. For $n^{-q / 2} \sum_{t=1}^{n}\left|x_{t}\right|^{-q}$, the same reasoning can be followed.

We are now ready to prove the main result of the paper.

Proof of Theorem 3. Define

$$
\begin{aligned}
X_{n} & =n^{-q / 2} \sum_{t=1}^{n} x_{t}^{-q} I\left(x_{t} \geq 0\right) ; \\
M_{n}(K) & =\sum_{t=1}^{n} I\left(0 \leq x_{t} \leq K n^{-1 / 2}\right) ; \\
X_{n K}^{(1)} & =n^{-q / 2} \sum_{t=1}^{n} x_{t}^{-q} I\left(0 \leq x_{t} \leq K n^{-1 / 2}\right)=\sum_{t=1}^{M_{n}(K)} Y_{n t}^{-q} I\left(0 \leq Y_{n t} \leq K\right) ; \\
X_{n K m}^{(2)} & =\sum_{t=1}^{\min \left(m, M_{n}(K)\right)} Y_{n t}^{-q} I\left(0 \leq Y_{n t} \leq K\right) ; \\
X_{n m}^{(3)} & =\sum_{t=1}^{\min \left(m, M_{n}(\infty)\right)} Y_{n t}^{-q} ; \\
X_{m}^{(4)} & =\sum_{t=1}^{m} Y_{t}^{-q} ;
\end{aligned}
$$

and consider the Laplace transform $E \exp \left(-r X_{n}\right)$ for $r>0$. We will first show that this Laplace transform converges by considering $E \exp \left(-r X_{n}\right)-E \exp \left(-r X_{n K}^{(1)}\right)$, $E \exp \left(-r X_{n K}^{(1)}\right)-E \exp \left(-r X_{n K m}^{(2)}\right), E \exp \left(-r X_{n K m}^{(2)}\right)-E \exp \left(-r X_{n m}^{(3)}\right)$, and $E \exp \left(-r X_{n m}^{(3)}\right)-$ $E \exp \left(-r X_{m}^{(4)}\right)$. For the first term, note that for all $r>0$, because $|\exp (-x)-\exp (-y)| \leq$ $|x-y|$ for $x, y \geq 0$,

$$
\begin{aligned}
& \limsup _{n \rightarrow \infty}\left|E \exp \left(-r X_{n}\right)-E \exp \left(-r X_{n K}^{(1)}\right)\right| \leq r \limsup _{n \rightarrow \infty} E\left|X_{n}-X_{n K}^{(1)}\right| \\
& \quad \leq r \limsup _{n \rightarrow \infty} n^{-q / 2} \sum_{t=1}^{n} t^{-q / 2} E\left(t^{-1 / 2} x_{t}\right)^{-q} I\left(t^{-1 / 2} x_{t}>t^{-1 / 2} n^{-1 / 2} K\right) \\
& \quad=r \limsup _{n \rightarrow \infty} n^{-q / 2} \sum_{t=1}^{n} t^{-q / 2} \int_{-\infty}^{\infty} f_{t}(x) x^{-q} I\left(x>t^{-1 / 2} n^{-1 / 2} K\right) d x \\
& \quad \leq r \sup _{t \geq 1, x \in \mathbb{R}} f_{t}(x) \limsup _{n \rightarrow \infty} n^{-q / 2} \sum_{t=1}^{n} t^{-q / 2}(q-1)^{-1}\left(t^{-1 / 2} n^{-1 / 2} K\right)^{1-q}
\end{aligned}
$$




$$
\leq C K^{1-q} \limsup _{n \rightarrow \infty} n^{-1 / 2} \sum_{t=1}^{n} t^{-1 / 2}=2 C K^{1-q} .
$$

To deal with the second term, note that $X_{n K}^{(1)} \neq X_{n K m}^{(2)}$ if $m<M_{n}(K)$, and therefore,

$$
\begin{aligned}
& \limsup _{n \rightarrow \infty}\left|E \exp \left(-r X_{n K}^{(1)}\right)-E \exp \left(-r X_{n K m}^{(2)}\right)\right| \\
& \quad=\limsup _{n \rightarrow \infty}\left|E\left(\exp \left(-r X_{n K}^{(1)}\right)-\exp \left(-r X_{n K m}^{(2)}\right)\right) I\left(X_{n K}^{(1)} \neq X_{n K m}^{(2)}\right)\right| \\
& \quad \leq 2 \limsup _{n \rightarrow \infty} P\left(X_{n K}^{(1)} \neq X_{n K m}^{(2)}\right) \\
& \quad \leq 2 \limsup _{n \rightarrow \infty} P\left(M_{n}(K)>m\right) \\
& \quad \leq 2 m^{-1} \limsup _{n \rightarrow \infty} E \sum_{t=1}^{n} I\left(0 \leq x_{t} \leq K n^{-1 / 2}\right) \\
& \quad \leq 2 m^{-1} \limsup _{n \rightarrow \infty} \sum_{t=1}^{n}\left(F_{t}\left(t^{-1 / 2} K n^{-1 / 2}\right)-F_{t}(0)\right) \leq C m^{-1} K
\end{aligned}
$$

where the third inequality is the Markov inequality and the last inequality follows from the mean value theorem and $\sup _{t \geq 1, x \in \mathbb{R}} f_{t}(x)<\infty$. Also, defining a summation over an empty index set as 0 , we have

$$
\begin{aligned}
& \left|X_{n K m}^{(2)}-X_{n m}^{(3)}\right| \\
& \quad=\left|\sum_{t=1}^{\min \left(m, M_{n}(K)\right)} Y_{n t}^{-q} I\left(0 \leq Y_{n t} \leq K\right)-\sum_{t=1}^{\min \left(m, M_{n}(\infty)\right)} Y_{n t}^{-q}\right| \\
& \quad=\left|\sum_{t=1}^{\min \left(m, M_{n}(K)\right)} Y_{n t}^{-q}-\sum_{t=1}^{\min \left(m, M_{n}(\infty)\right)} Y_{n t}^{-q}\right|=\sum_{t=\min \left(m, M_{n}(K)\right)+1}^{\min \left(m, M_{n}(\infty)\right)} Y_{n t}^{-q} \\
& \quad \leq \sum_{t=M_{n}(K)+1}^{M_{n}(\infty)} Y_{n t}^{-q}=\sum_{t=M_{n}(K)+1}^{M_{n}(\infty)} Y_{n t}^{-q} I\left(Y_{n t}>K\right) \\
& \quad \leq n^{-q / 2} \sum_{t=1}^{n} x_{t}^{-q} I\left(x_{t}>n^{-1 / 2} K\right)=\left|X_{n}-X_{n K}^{(1)}\right|,
\end{aligned}
$$

where the first inequality holds because if $m \leq M_{n}(K)$, then $m \leq M_{n}(K) \leq M_{n}(\infty)$ and the summation equals 0 . Therefore, we can follow the earlier argument for the first term to find $\limsup _{n \rightarrow \infty}\left|E \exp \left(-r X_{n K m}^{(2)}\right)-E \exp \left(-r X_{n m}^{(3)}\right)\right| \leq 2 C K^{1-q}$.

Also, note that because the random walk is recurrent, $M_{n}(\infty)$ will exceed any $m$ eventually, implying that eventually, $X_{n m}^{(3)}=\sum_{t=1}^{m} Y_{n t}^{-q}$. Therefore, because $\left(Y_{n 1}^{-1}, \ldots, Y_{n m}^{-1}\right)^{\prime}$ converges in distribution to $\left(Y_{1}^{-1}, \ldots, Y_{m}^{-1}\right)^{\prime}$ by Theorem 2, it follows that $X_{n m}^{(3)}$ converges in 
distribution to $X_{m}^{(4)}$. Putting all these results together, we now find $\limsup _{n \rightarrow \infty}\left|E \exp \left(-r X_{n}\right)-E \exp \left(-r X_{m}^{(4)}\right)\right| \leq 2 C K^{1-q}+C m^{-1} K+2 C K^{1-q}+0$.

Next, note that $\psi(r)=\lim _{m \rightarrow \infty} E \exp \left(-r X_{m}^{(4)}\right)$ is well-defined because $X_{m}^{(4)}$ is increasing in $m$. Therefore, for all $K>0$ and $m \geq 1$

$$
\begin{aligned}
& \limsup \left|E \exp \left(-r X_{n}\right)-\psi(r)\right| \\
& \quad \leq \limsup _{n \rightarrow \infty}\left|E \exp \left(-r X_{n}\right)-E \exp \left(-r X_{m}^{(4)}\right)\right|+\left|E \exp \left(-r X_{m}^{(4)}\right)-\psi(r)\right| \\
& \leq C m^{-1} K+4 C K^{1-q}+\left|E \exp \left(-r X_{m}^{(4)}\right)-\psi(r)\right| .
\end{aligned}
$$

Now taking the limit first as $m \rightarrow \infty$ and then $K \rightarrow \infty$, it follows that $\lim _{n \rightarrow \infty} E \exp \left(-r X_{n}\right)=$ $\psi(r)$. By Theorem 2 of Feller (1971) on page 431, $X_{n}$ now converges in distribution if $\lim _{r \downarrow 0} \psi(r)=1$. Note that

$$
\begin{aligned}
& \lim _{r \downarrow 0} \limsup _{n \rightarrow \infty}\left|E \exp \left(-r X_{n}\right)-1\right| \\
& \quad \leq \limsup _{K \rightarrow \infty} \lim _{r \downarrow 0} \limsup _{n \rightarrow \infty}\left|E\left(\exp \left(-r X_{n}\right)-1\right)\left(I\left(\left|X_{n}\right| \leq K\right)+I\left(\left|X_{n}\right|>K\right)\right)\right| \\
& \quad \leq \limsup _{K \rightarrow \infty} \lim _{r \downarrow 0} \limsup _{n \rightarrow \infty}\left(|r| E\left|X_{n}\right| I\left(\left|X_{n}\right| \leq K\right)+P\left(\left|X_{n}\right|>K\right)\right),
\end{aligned}
$$

and therefore it suffices to show

$$
\limsup _{K \rightarrow \infty} \limsup _{n \rightarrow \infty} P\left(\left|X_{n}\right|>K\right)=0
$$

$K \rightarrow \infty \quad n \rightarrow \infty$

that is, it suffices to show $X_{n}=O_{p}(1)$, which follows from Lemma 19 .

To show that $n^{-q / 2} \sum_{t=1}^{n}\left|x_{t}\right|^{-q} \stackrel{d}{\longrightarrow} \sum_{t=1}^{\infty} Z_{t}^{-q}$, we can reason analogously by defining

$$
\begin{aligned}
X_{n} & =n^{-q / 2} \sum_{t=1}^{n}\left|x_{t}\right|^{-q} ; \\
M_{n}(K) & =\sum_{t=1}^{n} I\left(\left|x_{t}\right| \leq K n^{-1 / 2}\right) ; \\
X_{n K}^{(1)} & =n^{-q / 2} \sum_{t=1}^{n}\left|x_{t}\right|^{-q} I\left(\left|x_{t}\right| \leq n^{-1 / 2} K\right)=\sum_{t=1}^{M_{n}(K)} Z_{n t}^{-q} I\left(Z_{n t} \leq K\right) ; \\
X_{n K m}^{(2)} & =\sum_{t=1}^{\min \left(m, M_{n}(K)\right)} Z_{n t}^{-q} I\left(Z_{n t} \leq K\right) ; \\
X_{n m}^{(3)} & =\sum_{t=1}^{\min (m, n)} Z_{n t}^{-q} ; \\
X_{m}^{(4)} & =\sum_{t=1}^{m} Z_{t}^{-q} .
\end{aligned}
$$




\section{REFERENCES}

Akonom, J. (1993) Comportement asymptotique du temps d'occupation du processus des sommes partielles. Annales de l'I.H.P. Probabilités et Statistiques 29, 57-81.

Berkes, I. \& L. Horváth (2006) Convergence of integral functionals of stochastic processes. Econometric Theory 22, 304-322.

Borodin, A.N. \& I.A. Ibragimov (1995) Limit theorems for functionals of random walks. In Proceedings of the Steklov Institute of Mathematics, vol. 195. American Mathematical Society.

Christopeit, N. (2009) Weak convergence of nonlinear transformations of integrated processes: the multivariate case. Econometric Theory 25, 1180-1207.

Chung, K.L. (2001) A Course in Probability Theory, 3rd Edition. Academic Press.

de Jong, R.M. (2004) Addendum to "Asymptotics for nonlinear transformations of integrated time series". Econometric Theory 20, 627-635.

de Jong, R.M. \& C.H. Wang (2005) Further results on the asymptotics for nonlinear transformations of integrated time series. Econometric Theory 21, 413-430.

Feller, W. (1971) An Introduction to Probability Theory and Its Applications, 2nd Edition. vol. II. John Wiley \& Sons, Inc.

Fréchet, M. \& J. Shohat (1931) A proof of the generalized second-limit theorem in the theory of probability. Transactions of the American Mathematical Society 33, 533-543.

Jeganathan, P. (2004) Convergence of functionals of sums of r.v.s to local times of fractional stable motions. The Annals of Probability 32, 1771-1795.

Lin, G.D. (2017) Recent developments on the moment problem. Journal of Statistical Distributions and Applications 4, 1-17.

Michel, J. \& R.M. de Jong (2020) The sum of the reciprocal of the random walk. Econometric Theory $36,170-183$.

Miller, K.S. \& B. Ross (1993) An Introduction to the Fractional Calculus and Fractional Differential Equations. Wiley.

Park, J.Y. \& P.C.B. Phillips (1999) Asymptotics for nonlinear transformations of integrated time series. Econometric Theory 15, 269-298.

Pötscher, B.M. (2004) Nonlinear functions and convergence to Brownian motion: beyond the continuous mapping theorem. Econometric Theory 20, 1-22.

Pötscher, B.M. (2013) On the order of magnitude of sums of negative powers of integrated processes. Econometric Theory 29, 642-658.

Van der Vaart, A.W. (2000) Asymptotic Statistics. Cambridge University Press. 